\title{
ANÁLISIS DEL PRIMER INTENTO DE IMPONER AUTORREGULACIÓN SOBRE GOBIERNO CORPORATIVO EN CHILE: RESULTADOS DE UN OXÍMORON
}

\author{
ANALYSIS OF THE FIRST ATTEMPT AT IMPOSING SELF REGULATION \\ ON CORPORATE GOVERNANCE IN CHILE: RESULTS OF AN OXYMORON
}

\author{
María Paz Godoy Uson* \\ EDUARDo WaLKer HitsChFELD** \\ Matías Zegers Ruiz-TAgLE ***
}

\begin{abstract}
RESUMEN: Entre los años 2012 y 2014, con el declarado propósito de fomentar la autorregulación, la Superintendencia de Valores y Seguros exigió a los emisores en Chile responder un cuestionario relacionado a prácticas de gobierno corporativo en la modalidad "comply or explain”. El estudio analiza las respuestas de 97 emisores para cada uno de estos años. El estudio primero verifica si las prácticas evaluadas por esta norma son consideradas "útiles", es decir, si se adoptan y si hubo cambios o evolución en las respuestas a través del tiempo. La segunda parte del estudio analiza si las respuestas fueron explícitas e informativas. Aunque las respuestas difieren según categoría, en promedio $65 \%$ de las prácticas fueron adoptadas, de las cuales un 59\% habrían sido adoptadas con anterioridad a la norma. Asimismo, la información en las respuestas tiende a ser estandarizada y escasa, entendiéndose como un tipo de cumplimiento formal y no necesariamente cualitativo de la Norma. Las respuestas evidencian escasos cambios en las prácticas de gobierno corporativo como consecuencia de la dictación de la Norma.
\end{abstract}

Palabras clave: Gobierno Corporativo, Autorregulación, Mercados Emergentes, NCG 341, Deberes Fiduciarios.

ABSTRACT: Between 2012 and 2014 the regulator issued a Norm requiring issuers to answer a questionnaire related with corporate governance practices in a "comply or explain" mode. This article studies the answers given by 97 issuers for those years. This article first verifies whether the practices included in the questionnaire are considered "useful", this is, if they were adopted andl or whether the answers evolved through time. Then, the article analyzes whether the answers were explicit and informative. Although there are differences between categories, on average $65 \%$ of the practices evaluated were adopted, of which 59\% were adopted before the Norm. In addition, the

\footnotetext{
* PhD (c) Maastricht University, Coordinadora de Investigación Centro de Gobierno Corporativo UC. Dirección postal: Avenida Libertador Bernardo O’Higgins 340, Edificio Derecho, Piso 4, Santiago, Chile. Dirección electrónica: magodoyu@uc.cl

** PhD UC Berkeley, Profesor Titular Escuela de Administración P. Universidad Católica de Chile, Director Centro de Gobierno Corporativo UC, Dirección postal: Avenida Vicuña Mackenna 4860, San Joaquín, Santiago, Chile. Dirección electrónica: ewalker@uc.cl

*** LL.M. The University of Michigan, Profesor Asociado Facultad de Derecho P. Universidad Católica de Chile, Presidente Centro de Gobierno Corporativo UC. Dirección postal: Avenida Libertador Bernardo O’Higgins 340, Edificio Derecho, Piso 4, Santiago, Chile. Dirección electrónica: mzegers@uc.cl Los autores agradecen la ayuda de Maria Jose Alliende, Carla Meza, Sebastián Yáñez y del Centro de Gobierno Corporativo UC. Cualquier error es propio.
} 
information contained in the answers seems to be standardized and scarce, suggesting formal and not necessarily actual compliance. Questionnaire answers also indicate few reported changes in governance practices as a consequence of this Norm.

Keywords: Corporate Governance, Self-Regulation, Emerging Markets, Chile, NCG 341, Fiduciary Duties.

\section{INTRODUCCIÓN}

En 2012 la Superintendencia de Valores y Seguros (“SVS”) emitió el primer marco normativo que pretendía elevar los estándares de gobierno corporativo de las sociedades anónimas abiertas en Chile ("Sociedades"). Esta iniciativa, plasmada en la Norma de Carácter General N³41 del año 2012 ("NCG 341" o "Norma”), abrió un debate frente a la definición y efectividad del conjunto de estándares definidos por la SVS, esencialmente radicados en el marco de actuación y profesionalización de los directorios.

En junio de 2015 la SVS derogó la Norma y emitió la Norma de Carácter General No 385 ("NCG 385”). La nueva normativa -actualmente vigente- consideró necesario ampliar de 19 a 99 las prácticas, con especial énfasis en el rol de control por parte del directorio y en la incorporación de estándares en materia de responsabilidad social y sustentabilidad.

Con ocasión de la derogación de la Norma y la emisión de la NCG 385, resulta útil analizar el impacto de la Norma en generar estándares de buenas prácticas en materia de gobierno corporativo en Chile ("Prácticas de GC"). En definitiva ¿̨logró la Norma mejorar los estándares de gobierno corporativo? Se busca responder esta interrogante a partir del análisis de las respuestas de 97 Sociedades del índice bursátil IGPA a las preguntas de la Norma durante su vigencia.

En particular, se intenta responder las siguientes preguntas: a) ¿ Significó la Norma un avance relevante en términos de autorregulación referida a las Prácticas de GC establecidas por la SVS, las que antes no existían o no necesariamente eran públicas?; b) ¿Contestaron las Sociedades el cuestionario por convicción o por obligación?; c) Las Sociedades que no adoptaron las prácticas, ¿`cómo se justificaron?; d) ¿Hubo prácticas que la Norma no consideró y que si debieron ser incorporadas?; y e) ¿Existió una evolución con cambios significativos a partir de la Norma?

El estudio se divide de la siguiente forma: en la primera sección analiza el desarrollo normativo del gobierno societario en Chile y las implicancias de ajustarse a un tipo de regulación bajo la modalidad comply or explain. La segunda sección busca determinar el impacto de la Norma en Sociedades pertenecientes al IGPA durante su vigencia. Los resultados se basan en dos análisis complementarios a nivel general y agregado. En primer lugar un análisis cuantitativo, que analiza el grado de cumplimiento con las prácticas de la Norma y además busca determinar la existencia de cambios significativos en las respuestas para concluir si estos cambios evolucionaron hacia un proceso de adopción o de no adopción. En segundo lugar un análisis cualitativo que describe y analiza las respuestas con el propósito de concluir si estas otorgaron información relevante al mercado. Por último, en la tercera sección se exponen las principales conclusiones. 


\section{REGULACION EN CHILE}

\section{1. ¿UUN SISTEMA CORPORATIVO EN EVOLUCIÓN O TRANSICIÓN?}

El sistema normativo que sustenta el gobierno corporativo de las Sociedades ha sido diseñado en gran parte por organismos estatales a través de leyes y regulaciones, las que a su vez han sido condicionadas por determinados eventos políticos y económicos. La historia parece ser un elemento clave a considerar; en especial si se quiere dar respuesta al tipo de desarrollo normativo impulsado por la autoridad. En particular, según un estudio del año 2000, el desarrollo normativo de las empresas chilenas y su mercado de capitales fueron una respuesta a episodios de crisis y otros eventos tales como la depresión financiera, las privatizaciones, la crisis de la deuda en 1982 y la reforma al sistema de pensiones ${ }^{1}$. Así, la necesidad por normar la actividad de las empresas y el mercado de capitales en Chile ha sido un proceso reactivo ante determinados sucesos. A esto, hoy podría sumarse otro elemento, un nuevo tipo de regulación está emergiendo - ex ante- para dar cabida a lo que según la SVS se debe entender como el estándar mínimo para un buen gobierno corporativo y el rol que le compete al directorio en la materia. Tomando en cuenta los efectos de una norma sobre la calidad de gobierno corporativo en las empresas es oportuno plantear las implicancias del tipo de regulación que ha impulsado la SVS².

En este contexto surge el primer intento por parte de la SVS de imponer autorregulación a las Sociedades mediante la emisión de la Norma. La responsabilidad de divulgar la información en ella requerida recayó en el directorio ${ }^{3}$, quienes por primera vez se vieron expuestos a plantearse responsabilidades no contenidas en la ley. El principal propósito de la Norma fue que las Sociedades informen al mercado sobre el cumplimiento de los estándares en ella establecidos. Con esto, se podría inferir que de lograr su propósito, la Norma modificaría las Prácticas de GC y la gestión de la información entregada al mercado. Encontrándonos aún en un proceso de reflexión sobre la pertinencia de estándares de gobierno corporativo impuestos por el regulador, resulta interesante rescatar los antecedentes que motivaron el surgimiento de la Norma. En definitiva, ¿cuál es el déficit que buscó llenar la Norma?

El marco jurídico que rige a las empresas y el mercado de capitales se encuentra en la Ley de Sociedades Anónimas No 18.045 y la Ley de Mercado de Valores No18.046, ambas de 1981. Estas leyes tuvieron el propósito de impulsar principios de libertad económica y establecer un rol subsidiario del Estado en la actividad empresarial. En principio, lo que se hizo fue dejar atrás un sistema de control estatal para dar paso a uno orientado al accionis$\mathrm{ta}^{4}$. Ambas leyes y sus posteriores reformas incorporaron principios inspirados en el sistema Anglosajón -siendo que el ordenamiento chileno se rige por el sistema civil francés- dificultando con esto la tarea de interpretar el espíritu de la ley ${ }^{5}$. En un comienzo, el conoci-

\footnotetext{
LEFORT Y WALKER (2000).

2 Lefort y GonZÁlez (2008) p. 19.

3 La NCG N³41 señala: "El directorio (...) será responsable de exponer en forma correcta, precisa, clara y resumida la manera en que ha adoptado cada práctica”.

4 NúNÉEZ y Pardow (2010) p. 237.

5 Lefort y Walker (2000) p.199.
} 
miento de la calidad del gobierno corporativo de las empresas fue de difícil acceso, ya que estas adoptaron los requerimientos mínimos normativos y de entrega de información a sus inversionistas ${ }^{6}$. La divulgación más detallada se dio posteriormente, tales como la descripción de los negocios y resultados, los factores de riesgo, sociedades relacionadas y otros aspectos relevantes con el fin de proveer mayor información al mercado 7 .

Es comprensible que las siguientes reformas legislativas hayan sido condicionadas a la estructura de propiedad que presentan las empresas en Chile, con alto nivel de concentración, control directo o indirecto por un grupo económico ${ }^{8}$, y estructuras piramidales como común denominador en el mercado, lo que genera intereses contrapuestos entre el accionista mayoritario y el minoritario. Es por esto que las reformas legislativas han tenido un especial interés por proteger al accionista minoritario.

La primera de ellas ocurrió el año 2000 mediante la Ley 19.705. Conocida como la Ley de OPAS, esta tuvo el propósito de ampliar la protección de los intereses de los accionistas minoritarios en procesos de cambio de control ${ }^{9}$. Entre otras cosas, se introdujo la figura del director independiente y el comité de directores. Hay que recordar que en ese tiempo Chile era parte del grupo de países que comenzaron a mejorar la calidad de su gobierno corporativo. A nivel regional se podía observar un avance en el sistema a partir de un desarrollo normativo como respuesta a escándalos corporativos y al interés que algunas organizaciones internacionales mostraban en el mercado de capitales regional ${ }^{10}$. Así, el foco del regulador regional estuvo en mejorar la protección del accionista minoritario y otros se sumaron a la iniciativa de generar códigos de gobierno corporativo (en adelante, "Códigos”) a partir del interés de los inversionistas (en 2007 la SVS se había mostrado interesada en sumarse a la iniciativa de elaborar un Código $)^{11}$. Tomando en cuenta que otros países en la región ya contaban con códigos propios, se estimó que la autorregulación era el camino correcto. Si bien Chile mantenía un liderazgo importante en la región, había preocupación por el mayor avance que otros países emergentes (como Malasia y Singapur) tenían a la fecha $^{12}$.

La segunda reforma ocurrió el 2009, mediante la Ley No $20.382^{13}$ que buscó perfeccionar los mecanismos de divulgación de información al mercado, adoptar políticas para prevenir el uso de información privilegiada, potenciar el funcionamiento del directorio e incorporar criterios de independencia para directores ${ }^{14}$. En ese entonces, el legislador tenía

\footnotetext{
Camus (2015).

Camus (2015).

8 LEForT Y WaLKer (2000).

9 La ley de OPAS fue consecuencia del caso Chispas, considerado caso de vulnerabilidad de los derechos de los accionistas minoritarios.

10 Lefort y Walker (2005) p. 72.

11 MCKINSEY E ICARE (2007).

12 MCKinsey e ICARE (2007).

3 WilenMANn (2010).

14 Asimismo, durante esta etapa la SVS impulsó distintos mecanismos para mejorar el gobierno corporativo, como la emisión de la NCG 211 instruyendo la confección de manuales de manejo y difusión de la información de interés para el mercado.
} 
una especial preocupación por disminuir los problemas de asimetría de información, agencia y riesgos de abuso ${ }^{15}$. Por ejemplo, se detectó que las mayores falencias estaban relacionadas con la transparencia y entrega de información al mercado ${ }^{16}$. Se señaló que era difícil formarse una opinión sobre el estado interno de la empresa, mientras que algunos agentes podrían acceder y utilizar información privilegiada ${ }^{17}$.

Pese a ello a dicha fecha se reconocía que existía un grado de cumplimiento razonable de los estándares de regulación en gobierno corporativo, considerándose incluso como uno de los más avanzados en la región ${ }^{18}$, con protección adecuada a los accionistas minoritarios, al nivel del Reino Unido y Estados Unidos $^{19}$ pero justificándose plenamente la necesidad de dichas reformas ${ }^{20}$. A esto se sumó que varias Sociedades tuvieron la iniciativa de emitir ADRs en EE.UU., elevando así sus estándares en virtud de la regulación en la materia, y a pesar de la alta concentración de propiedad existente, estudios internacionales situaban a Chile con un buen nivel de cumplimiento de principios de gobierno corporativo ${ }^{21}$. Prácticamente no existían sanciones administrativas producto de infracciones o incumplimientos por parte de los directores y la jurisprudencia era escasa, con lo que se confirmaba lo anterior ${ }^{22}$. Con todo, hay que recordar que gran parte de las reformas fueron parte del proceso de entrada de Chile a la OCDE el año 2010 lo que explicaría la necesidad por parte del legislador de hacer cambios legales ${ }^{23}$.

La percepción de la calidad del gobierno corporativo en Chile cambió una vez que se conocieron algunos escándalos. Lógicamente hubo un impacto en la opinión pública, y esto aceleró el trabajo de la SVS para lo que sería la primera normativa que, según sus objetivos, promovería la autorregulación. El regulador consideró necesaria la inmediata protección de la reputación y credibilidad del mercado ${ }^{24}$, mientras que los procesos judiciales y administrativos abrieron un debate sobre el rol del directorio, el cumplimiento de deberes fiduciarios teóricamente no esclarecidos en la ley y el tipo de responsabilidad que tienen por infracciones a esta, pero puede pensarse que estos fueron casos excepcionales y

15 LEY No $20.382(20 / 10 / 2009)$.

16 LeFort y GonZÁlez (2008).

17 LeFort y GonZÁlez (2008).

18 World Bank (2003). Ver también Lefort y González (2008) p. 8 y Alcalde (2009) p. 91.

19 Lefort y WaLker (2000).

20 HISTORIA DE LA LEY No 20.382 (2009). Se desprende del proyecto de ley que se encomienda a la SVS dictar NCG que contribuyan a la formulación y mejor funcionamiento de las mencionadas políticas. Ver MCKINSEY e ICARE (2007).

21 Hormazábal (2010). Ver también Mckinsey e ICARE 2004 y 2007. En 2004 Chile presentaba un 65\% de cumplimento de principios de buen gobierno definidos por la OECD (según scorecard de McKinsey aplicado a 20 empresas chilenas); uno de los más altos entre países emergentes. Este indicador global mostraba que las principales oportunidades de mejora era el Funcionamiento del Directorio, aunque existían en otras dos dimensiones ("Participación y Derechos de los Accionistas" y "Divulgación de Información y Transparencia"). Una nueva medición en 2007 a 19 empresas que representan sobre el 50\% de la capitalización bursátil, mostraba avances en el grado de cumplimiento.

22 LEFORT Y GONZÁLEZ (2008) p.7.

23 OECD (2011).

24 Pavez (2014). 
en ningún caso debieron entenderse como el reflejo de la calidad de gobierno corporativo en Chile ${ }^{25}$.

En este sentido, unos de los aspectos fundamentales de gobierno corporativo en Chile son los conflictos de intereses que surgen en las empresas nacionales comúnmente asociados al alto nivel de concentración de propiedad en manos de un grupo reducido de accionistas $^{26}$. En razón de esto, es válido poner especial atención en casos como las transferencias de control, transacciones entre partes relacionadas o la apropiación de oportunidades de negocios, por cuanto el deber de lealtad podría verse mayormente vulnerado. Sin embargo, cuando se trata del deber de cuidado, los directores tienen mayores incentivos para cumplir con su labor de manera diligente por cuanto el accionista controlador, al tener un interés real en el negocio, supervisará de cerca la labor del directorio ${ }^{27}$. En la práctica, esto implicaría un menor número de casos por infracciones al deber de cuidado que infracciones al de lealtad. Por ello, es interesante plantear cuál era la necesidad de regular el deber de cuidado de forma tan estricta como lo hizo la Norma y su sucesora.

Al observar los Códigos de países más avanzados y con estructuras de propiedad similares a la chilena, la calidad del gobierno corporativo no depende de las tareas de supervisión o control que ejerce el directorio (como se desprende de la Norma). Por el contrario, el directorio tiene un adecuado grado de discrecionalidad entre la labor de control y la labor estratégica de crear valor. Más interesante aún es que los accionistas, en particular, los mayoritarios, son considerados como un elemento de gobierno positivo en función de su rol supervisor. Por ejemplo, en el Código sueco hay un especial reconocimiento al accionista mayoritario "...Swedish society takes a positive view of major shareholders taking particular responsibility for companies by using seats on boards of directors to actively influence governance..."28. Otros, como el Código holandés hace un llamado al accionista de forma más amplia: "The general meeting should be able to exert such influence on the policy of the management board and the supervisory board of the company that it plays a fully-fledged role in the system of checks and balances in the company". En este sentido, resulta cuestionable que la Norma condicione la calidad del gobierno corporativo casi exclusivamente al directorio, ya que hay un desbalance mejorable mediante un refuerzo del rol del accionista ${ }^{29}$. Por lo demás, en el caso de Chile, este desbalance se aminora a partir de la participación de inversionistas institucionales, fundamentalmente las AFPs, en su calidad de minoritarios quienes con su intervención habrían permitido mejorar los mecanismos internos de gobierno societario.

\footnotetext{
25 Barclay (2015).

26 Alcalde (2013) p. 92.

27 Alcalde (2013) p. 92.

28 Swedish Corporate Governance Code (2015) p. 7. También Dutch Corporate Governance Code (2009) p. 7.

29 En Chile hay evidencia estadística sobre el aporte del sistema de AFP al desarrollo del mercado de capitales. Corbo y Schmidt-Hebbel (2003) y Lefort y Walker (2002).
} 


\subsection{PRimer intento Formal De PROMOVER La AUTORREGUlación}

La Norma incentivó formalizar públicamente diversas prácticas que probablemente ya se adoptaban con anterioridad. Es decir, si bien la autorregulación en la materia era inexistente hasta 2012, bien pudieron las Sociedades desde antes incorporar Prácticas de GC sin que estas hayan sido informadas al público ${ }^{30}$. En algunos países el desarrollo normativo en la materia ha sido liderado por entidades no gubernamentales. Este no ha sido el caso chileno, donde las iniciativas privadas de autorregulación no han prosperado, a juicio del regulador, por una falta de sentido de urgencia ${ }^{31}$. Con esto, la supuesta pasividad del sector privado habría acelerado un proceso de regulación que culminó en la Norma. En Julio de 2012 la SVS puso en consulta al público el proyecto de norma y el 29 de noviembre del mismo año la emitió, recogiendo un conjunto de 19 estándares y prácticas complementarias al marco jurídico vigente ${ }^{32}$. La novedad de la Norma fue que estableció obligaciones para la difusión de información bajo la modalidad de comply or explain.

Cabe reconocer que la SVS ha estado especialmente interesada en el grado de desarrollo internacional en la materia. Hoy en día muchos países cuentan con nuevas versiones de Códigos, que recogen principios y prácticas que en su mayoría han sido importados de otros sistemas más avanzados y que por lo general convergen en el ampliamente difundido principio comply or explain. Gran parte de este desarrollo internacional fue influido por escándalos financieros como Enron y WorldCom, y en especial por los efectos de la crisis financiera del 2007-2008, donde el rol secundario del directorio fue fuertemente criticado ${ }^{33}$. La motivación del regulador de elevar los estándares de gobierno corporativo proviene de la necesidad, en su opinión, de fortalecer la figura del directorio como el principal órgano de gobierno en su calidad de supervisor de la gestión. Sin embargo, más que atribuciones, el regulador optó por requerir del directorio una mirada esencialmente controladora y de su-

30 CAmus (2015) señaló "A la mayor información incorporada a las memorias anuales y autoevaluaciones de adopción de prácticas de gobierno corporativo (...), las sociedades han adoptado las siguientes medidas para disponer mayor información (...): a) Desempeño de la acción de la compañía. b) Formulario 20-F (para aquellas sociedades listadas en EE.UU.). c) Información de los resultados históricos de la sociedad. d) Reporte de sostenibilidad. e) Códigos de Conducta y Gobierno Corporativo. f) Información de las juntas de accionistas g) Calendarios de eventos corporativos. h) Video conferencias para la explicación de los resultados de la compañía, entre otros".

31 Coloma (2012).

32 SVS (2015). Proyecto Normativo SVS Junio 2015 p. 4 “...La premisa sobre la cual la SVS escogió la solución contenida en la Norma de Carácter General N³41, fue que al difundir información sobre las prácticas adoptadas por las sociedades anónimas abiertas: i) los inversionistas estarían en condiciones de exigir a las mismas contar con gobiernos corporativos robustos y eficaces que garanticen que su inversión será gestionada de la mejor forma posible o, de lo contrario, no invertirían en dichas sociedades; ii) otros actores del mercado, como es el caso de las bolsas de valores, centros de gobierno corporativo o universidades, podrían generar de forma agregada diversos análisis o estudios que también sirvan como antecedentes para la toma de decisiones de los inversionistas y como referencia para el resto del mercado; iii) los accionistas minoritarios, podrían conocer cómo está siendo gestionada la entidad en la que participan; y iv) que tanto los directores como los accionistas contaran con elementos objetivos, como lo son las prácticas adoptadas por sus competidores, proveedores u otras entidades para resguardar el interés social de la entidad, para discutir, cuestionar o proponer diversos temas de gobierno corporativo al interior de la sociedad".

33 Clarke (2011) pp. 28-49. 
pervisión. Este enfoque es contrapuesto a lo que se observa en otros Códigos, donde existe primero un reconocimiento a la labor estratégica del directorio de crear valor ${ }^{34}$ (Reino Unido, Suiza, Holanda y Malasia).

La Norma fue percibida como una oportunidad de mejora pero a la vez como poco relevante y confusa por el sector privado ${ }^{35}$. Algunos emisores señalaron que sus costos superarían los potenciales beneficios, mientras que otros la estimaron como un conjunto de prácticas pertinentes y como un tipo de cuestionamiento sano al interior de los directo$\operatorname{rios}^{36}$. Según el regulador, la Norma fue "la respuesta a una falencia manifiesta en el tiempo" lo que se reflejó en sus pronunciamientos asociados a procesos sancionatorios u otros. Se consideró que había falencias en el gobierno corporativo en materias como el deber de cuidado e información, y en lo relativo a las operaciones entre partes relacionadas ${ }^{37}$.

La definición de estándares mínimos de actuación permitiría mitigar casos de conflictos entre los distintos agentes y a su vez mejorar el funcionamiento de las empresas en el largo plazo. En principio la Norma nace como un intento por resolver cuál debe ser el actuar del directorio. De ser así, se debió haber logrado entregar certidumbre sobre "qué es lo debido" por parte de un director ${ }^{38}$. Sin embargo, el riesgo implícito en la definición no vinculante de estándares es que al no existir una conducta tipificada en la ley sobre la labor de los directores, los tribunales recurrirán a los estándares que ha establecido la SVS para evaluar en concreto la diligencia debida. Ante esto cabe preguntarse si corresponde que los jueces al momento de fallar cuenten con lo que a juicio de la SVS se debe entender por buen gobierno corporativo, siendo que es la opinión del regulador al respecto, y siendo además que al menos actualmente la misma SVS es quien formula cargos y sanciona en caso que considere que han violado las normas que el impone.

Así, los casos nacionales relacionados con gobierno corporativo, por lo general han surgido a partir de cuestionamientos a nivel ejecutivo y/u operacional, en donde los procesos administrativos y/o judiciales han terminado por responsabilizar a los directores en virtud del incumplimiento de sus deberes de lealtad y cuidado. Casos como La Polar, D\&S, FASA han abierto el debate sobre el grado de responsabilidad que le compete al directorio

\footnotetext{
34 Uk Corporate Governance Code (2014) p.7 Section A: Leadership A.1: The Role of the Board: Main Principle: "Every company should be headed by an effective board which is collectively responsible for the long-term success of the company"... "The board's role is to provide entrepreneurial leadership of the company within a framework of prudent and effective controls which enables risk to be assessed and managed." Ver también DUTCH CORporate Governance Code (2009) p. 11; Swedish Corporate Governance Code (2015) p. 16; y Malasyan CODE ON CORPORATE GOVERnANCE (2012) p. 5 “...In discharging its fiduciary duties and leadership functions, it is imperative for the board to govern and set the strategic direction of the company while exercising oversight on management...".

35 La Tercera (21/04/2013).

36 La Tercera (21/04/2013).

37 Coloma (2012).

38 Se ha señalado que una de las principales complejidades del sistema chileno es la indeterminación del comportamiento en cuanto al deber de cuidado. La LSA contempla un concepto jurídico indeterminado, por tanto la labor de los jueces resulta esencial para entregar un mayor grado de certeza. El problema es que los tribunales tampoco cuentan con una lex artis a la cual puedan recurrir para valorar el modelo de conducta debido por parte de los directores. Ante este escenario es difícil establecer ex ante cómo administrar diligentemente una empresa. Ver AlCalde (2013).
} 
en cuanto a la extensión de sus deberes fiduciarios. La dificultad está en que, al no establecerse con certeza cuál es el deber del directorio o la diligencia debida por este, las sociedades se ven expuestas a que sus directores incurran inadvertidamente en el incumplimiento de deberes.

\subsection{AUTORREGULACIÓN ¿¿FORZADA O VOLUNTARIA?}

A nivel comparado, el comportamiento de las empresas se ha tratado de normar mediante principios de manera no vinculante (principle-based approach) con la mayoría de los países siguiendo el modelo inglés. Por el contrario, en muy pocos países se aplica de manera estricta la ley (rules-based approach) como ocurre en Estados Unidos (ejemplo de sistema rígido amparado en sus leyes Sarbanes Oxley y Dodd Frank), India, Turquía, Israel y Arabia Saudita ${ }^{39}$. Ante esto, es razonable preguntarse si para el caso chileno corresponde que un modelo de autorregulación contemple principios específicos y rígidos que parecen regulación más que autorregulación. Por otro lado, hay que tomar en cuenta que un sistema de autorregulación puede bien ser el anticipo de regulación, es decir, que los estándares pasen a ser obligaciones como ha ocurrido en España, donde al modificar la Ley de Sociedades de Capital la Comisión de Expertos en materia de Gobierno Corporativo señaló que “...sin menospreciar el valor de las recomendaciones y del modelo de «cumplir o explicar", se considera necesario proponer cambios normativos relativos al gobierno de las empresas" 40 .

Siendo este el primer intento por desarrollar un modelo de gobierno corporativo no vinculante, parece necesario cuestionar si la autorregulación que promovió la SVS logró mejorar los estándares de gobierno corporativo. Así, se debe resolver si es aconsejable optar por establecer solo principios y objetivos generales de cumplimiento, o por el contrario, establecer principios tan específicos que podrían llegar a ser difícilmente alcanzables. En cierta medida, la Norma pudo ser una suerte de catálogo sobre procedimientos y políticas al interior de las empresas pero no contó previamente con la identificación de principios claros y generales que permitieran amparar cada práctica en particular, algo que sí se observa en Códigos extranjeros ${ }^{41}$. Es posible que las Sociedades, al no identificar o no entender el propósito detrás de cada práctica, no hayan tenido una motivación real por incorporarlas.

Si bien la SVS impulsó la Norma como autorregulación se trató de una normativa que exigió a todos los emisores informar sobre la adopción de prácticas establecidas por ella misma, lo que implica que lo desarrollado por la SVS ha sido regulación propiamente tal ${ }^{42}$.

\footnotetext{
39 OECD (2015).

40 Coglianese et al. (2004) p. 4 señalan “...Should regulatory standards articulate broad goals or purposes, guiding behavior through the adherence to general principles? Or should regulations take the form of specific rules that tell companies and their lawyers and auditors exactly what is acceptable and unacceptable? Rules have their virtues, and they have been widely used, but they also may allow corporate actors to find ways to comply with the letter of the law while circumventing its spirit".

41 Así, el UK CORPORATE GOVERNANCE CODE (2014) establece dos tipos de principios; unos generales y otros específicos. Las empresas deben informar cómo cumplen los principios generales y si han adoptado las prácticas más específicas, de lo contrario deben emitir una explicación por el no cumplimiento.

42 Zegers y Consiglio (2013).
} 
Incluso, la misma definición de "autorregulación" que establece la SVS da a entender que la Norma podría no considerarse como tal ${ }^{43}$, ya que no fue un proceso por el que un grupo de empresas se someten a reglas establecidas de común acuerdo. Más aún, siguiendo a la SVS, la normativa sería un tipo de sometimiento reglamentario forzado ${ }^{44}$ ya que “...por autorregulación debe entenderse el proceso mediante el cual un conjunto de entidades sin relaciones jerárquicas entre ellas..., se someten a reglas establecidas de común acuerdo y cuyo objeto es preservar la eficiencia y reputación del mercado, sector o industria a la que pertenecen. Si el sometimiento a dichas reglas es voluntario, la autorregulación se denomina voluntaria, en caso contrario, es forzada... "\$5

Con todo, y desde un punto de vista retórico, para algunos la autorregulación ha sido asociada a un oxímoron ${ }^{46}$ al unirse dos ideas de significación contradictoria. Así, resulta paradójico dejar a la parte regulada establecer sus propias reglas, y más en el caso de la NCG 341, donde pese a que es un formato de comply or explain, los emisores deben responder en forma obligatoria y las preguntas son respecto a aquellas materias que el regulador propone como buenas prácticas, y no las que proponen los mismos emisores ${ }^{47}$. Con esto se quiere decir que reconociendo la flexibilidad que invoca la autorregulación, para ser útil y/o eficaz debe necesariamente coincidir con las expectativas y realidades de las Sociedades, de lo contrario los esfuerzos por mejorar los estándares de Prácticas de GC será en vano.

\subsection{COMPLY OR EXPLAIN EN CHILE: ONE SIZE FITS ALL?}

El reconocimiento del principio comply or explain es un elemento innovador en nuestro ordenamiento jurídico. Su fin es otorgar flexibilidad en el nivel de cumplimiento, entendiéndose que las empresas deben cumplir con los estándares si se ajustan a sus realidades y circunstancias, manteniendo la autonomía y flexibilidad para estructurar la empresa y el funcionamiento de sus órganos de gobierno y adaptarlos a sus necesidades (estructura de propiedad, tamaño, especificaciones sectoriales, etc.). Por tanto, las Sociedades pueden reflexionar sobre sus prioridades en materia de gobierno corporativo, desarrollando un análisis crítico sobre cada estándar o práctica.

\footnotetext{
43 SVS (2015).

44 SVS (2015).

45 SVS (2015).

46 El diccionario de la Real Academia Española lo define como la “...combinación, en una misma estructura sintáctica, de dos palabras o expresiones de significado opuesto que originan un nuevo sentido..."

47 Coglianese, et al. (2004) p. 224 "...To some, the term self-regulation is an oxymoron, or something akin to the fox guarding the chicken coop". Ver IRWIN et al. (2012) p.1067 "Self-regulation has always presented the proverbial problem of the "fox guarding the henhouse." In the bright light of day, the fox dutifully guards his post. (...) Inevitably, the sun fades and the shadowy hand of night sweeps across the horizon. The fox abandons his post and slips surreptitiously inside the henhouse. He has no apprehension, no reason to look over his shoulder. He answers to no one. He makes his own rules. He is his own regulator, motivator, and enforcer. Such is the dilemma faced by any group governed by the pure principle of self-regulation".
} 
El principio se justifica en una sola frase muy difundida internacionalmente: "one size does not fit all'. Con ello se explica que las empresas son diferentes entre sí, incluso aquellas de un mismo tamaño e industria. Por tanto, pretender igualarlas o estandarizarlas mediante un conjunto de prácticas puede ser inviable y contraproducente. Utilizar el principio comply or explain como fórmula de cumplimiento puede ser útil si se evalúan prácticas alcanzables. En general, una normativa de estas características debiera apelar a la flexibilidad y no a la imposición de estándares difícilmente alcanzables o solo teóricos, ya que se podría argüir que con ello se llega a un tipo de cumplimiento formal y estandarizado

Existen estudios comparados que han tomado en cuenta mercados emergentes como Chile para explicar cómo mejorar el gobierno de una empresa mediante un conjunto de buenas prácticas. Un modelo de autorregulación se considera como una herramienta atractiva, pero es esencial considerar las características del país e incluso más las de las empre$\operatorname{sas}^{48}$. No es claro si la SVS tomó esto en cuenta antes de proponer la Norma.

\subsection{AVERSIÓN AL RIESGO DE INCUMPLIMIENTO}

Es posible decir que la Norma no goza de la legitimidad necesaria para mejorar los estándares de gobierno corporativo, al haber sido impuesta como regulación antes que ser fruto de la autorregulación. Un emisor no estaría dispuesto a entregar más información que el mínimo requerido legalmente para no exponerse innecesariamente a riesgos frente al regulador y al mercado. Es decir, es probable que las Sociedades que hayan generado cambios fueran por el riesgo legal asociado al no cumplimiento.

La preocupación por explicaciones poco satisfactorias es el punto débil de este sistema, lo cual ha sido advertido internacionalmente con anterioridad. La calidad de las explicaciones es algo que incluso en los países más avanzados se intenta mejorar. De hecho, han existido iniciativas para promover mejores explicaciones por parte de las empresas que deban dar cumplimiento a Códigos bajo el principio comply or explain. Así, la Comisión Europea emitió el 2014 el documento "Commission Recommendation on the quality of corporate governance reporting (comply or explain)" 49 . Asimismo, el Financial Reporting Council, regulador del Reino Unido emitió directrices al respecto bajo el documento "What constitutes an explanation under comply or explain?" de $2012^{50}$.

\section{6 ¿MERCADO MEJOR INFORMADO?}

La SVS señaló que la difusión pública de adoptar o no una práctica “... generaría los incentivos para elevar los estándares ya que permitiría a los inversionistas, proveedores, competidores, empleados y demás grupos de interés contar con esa información al momento de tomar una decisión de inversión (...) pudiendo privilegiar a aquellas sociedades en que sus intereses están mejor resguardados”. A juicio de algunos, la Norma lograría reducir

48 Black et al. (2012) pp. 934-937. También Black y KRAaKman (1996) p. 1914 "The law that works for a developed economy, when transplanted to an emerging economy, will not achieve a sensible balance among company managers' need for flexibility to meet rapidly changing business conditions (...). The defects in the law will increase the cost of capital and reduce its availability".

49 UE Commission Recommendation (2014).

50 UK Financial Reporting Council (2012). 
las asimetrías de información que existen en el mercado permitiendo que los inversionistas cuenten con valiosa información ${ }^{51}$.

Consideramos que con un cumplimiento formal de la Norma es difícil que este tipo de incentivos puedan generarse. De acuerdo a la experiencia comparada, el inversionista asocia el valor accionario con la calidad de gobierno corporativo, pero la realidad es que esto no siempre es así. La adopción del principio comply or explain también tiene desventajas relacionadas con el nivel de transparencia apropiado para la realidad de la empresa. Así, se ha señalado que empresas con bajo rendimiento económico que no elevan sus estándares de gobierno corporativo serían castigadas por el mercado sin que los inversionistas hayan evaluado las explicaciones a partir del principio comply or explain. Esto puede ocurrir incluso cuando la no adopción de una práctica se haya decidido en el mejor interés de la empresa $^{52}$. La consecuencia es que en el largo plazo se llega a un tipo de cumplimiento estandarizado y formal. Es decir, para evitar estos inconvenientes sería mejor informar al mercado que se cumple (formalmente) sin dar mayores explicaciones. En la práctica los inversionistas no leerían (o no comprenderían) las explicaciones por el no cumplimiento de una práctica, por lo que el costo de no cumplir podría ser alto.

Más aún, a partir de la información divulgada se generarían rankings o índices de calidad lo que resulta contraproducente si se reconoce que la información divulgada no es fielmente representativa de la realidad de cada empresa si se ignoran las explicaciones. La simple tabulación de resultados basados en porcentajes de cumplimiento carece de sentido e informar al mercado eventuales rankings es un análisis simplista de una realidad compleja. Por tanto, es inevitable preguntarse hasta qué punto este tipo de divulgación es útil al momento de tomar una decisión de inversión.

Asimismo, en el caso de las Sociedades, tener la propiedad concentrada no generaría los incentivos necesarios para elevar los estándares mediante la incorporación de nuevos inversionistas. En otras palabras el controlador cumpliría con la normativa solo por existir un requerimiento legal (los estándares son voluntarios pero las explicaciones no) y no por la oportunidad de atraer potenciales inversionistas u otros grupos de interés. Los intereses de los directores están alineados con los de los accionistas; esencialmente los controladores, quienes tienen los votos necesarios para elegirlos. Si bien la posición dominante de un accionista contribuye a un mayor grado de supervisión de la administración (reduciendo los conflictos de intereses "verticales"), esto a su vez puede terminar contribuyendo a aumentar los conflictos entre controladores y minoritarios ("horizontales") 53 .

Un reciente estudio elaborado por la OCDE en más de 40 países estableció que Chile es el único país que no exige divulgar el cumplimiento de Principios de GC en las memorias anuales ${ }^{54}$. Por lo tanto, sería difícil que los inversionistas o los grupos de interés puedan tomar conocimiento de manera fácil sobre su nivel de gobierno corporativo.

Finalmente, la autorregulación en esta materia es un desafío que merece un especial análisis. Hay que reconocer los esfuerzos realizados por la SVS hasta la fecha, los que

51 García (2014).

52 Enriques et al. (2009) p. 67 pie de página 65 en referencia a Hertig (2005) pp. 273-274. Ver también CoOmbes (2014) y Haskovec (2012) p. 11-12 y 17.

53 Véase Young et al. (2008).

54 OECD (2015). 
han generado un debate transversal entre los distintos actores del mercado interesados por mejorar el funcionamiento de las empresas. Eso sí, hay que tomar en cuenta que cualquier normativa de autorregulación en materias societarias debe ampararse en un marco legal claro y con una clara enunciación de sus principios fundamentales aceptados por las Sociedades que les permita un rango de actuación flexible y alcanzable, ya que de lo contrario terminará convirtiéndose en un mecanismo de cumplimiento formal de poca utilidad ${ }^{55}$.

\section{HIPÓTESIS}

Este estudio analiza diversas hipótesis referentes al impacto que habría tenido la Norma en las Sociedades. Se toma como hipótesis de trabajo que la SVS a través de la Norma habría dado un paso significativo en materia de autorregulación referida a Prácticas de GC, lo que debería reflejarse en las respuestas y en su evolución en el tiempo.

Por el contrario, al ser la Norma una suerte de autorregulación forzada (emanada del regulador), hipótesis alternativas son a) no generó cambios significativos en las respuestas; b) de haber generado cambios, estos se dieron por el riesgo legal asociado al no cumplimiento; c) no produjo información relevante a los inversionistas respecto de las Prácticas GC; y d) omitió Prácticas de GC que son necesarias para la obtención de buenos resultados en el largo plazo.

Para contrastar estas hipótesis contrapuestas, se analiza: i) qué proporción de las prácticas fue considerado relevante por las Sociedades y si hubo cambios significativos en la adopción de estas en el tiempo; ii) si las respuestas entregaron información útil para evaluar el gobierno corporativo, es decir, si fueron explícitas e informativas; y iii) si, a la luz de las respuestas, existían prácticas importantes que no fueron consideradas en la Norma.

\section{METODOLOGÍA}

La muestra la conforman Sociedades pertenecientes al IGPA durante el periodo de vigencia de la Norma. Para este estudio se realizaron dos tipos de análisis complementarios. En primer lugar, un análisis cuantitativo, que busca determinar el grado de adopción de prácticas y de cambios significativos en las respuestas, con el fin de concluir si estas evolucionaron hacia un proceso de adopción (cambio a positivo) o a no adopción (cambio a negativo). En segundo lugar, un análisis cualitativo sobre la calidad de la información divulgada en cada práctica, para concluir si fue relevante para los inversionistas.

La muestra resultó en 97 Sociedades IGPA durante los años 2012- 2014 (Anexo 1). Del total de respuestas emitidas por las Sociedades se analizaron solo aquellas que se encontraban disponibles en la página web de la SVS al 25 de septiembre de 2015, por lo que no se accedió a las respuestas de 15, dejando la muestra en 82 Sociedades. Considerando 19 prácticas, el número total de observaciones (número de prácticas multiplicado por el número de empresas) es 1.558. Cada una de las empresas contestó el mismo cuestionario todos los años.

55 En este sentido, es interesante ver PAZ-AREs (2004) quien postula que cualquier cambio relacionado al gobierno corporativo es necesariamente un esfuerzo de largo de plazo. 
Las respuestas emitidas por las Sociedades se analizaron a nivel agregado y correspondieron a las secciones enunciadas en la Norma.

\subsection{METODOLOGÍA DE ANÁLISIS CUANTITATIVO}

La primera parte busca determinar a través de un test estadístico de proporciones si cada práctica es estadísticamente significativa (Anexo 3). Este es un concepto estadístico que resume las respuestas de adopción reportadas por las Sociedades durante el período de muestra (con un valor de 1 si se adopta y 0 si no lo hace), lo que no permite obtener conclusiones en esta parte sobre la eficacia o calidad de cada práctica. La significancia estadística de una práctica en particular no resuelve la incertidumbre clínica ocurrida durante el periodo de análisis. En definitiva esta parte pretende concluir (i) si a partir de los cambios observados en las respuestas la Norma generó un impacto extrapolable a todas las Sociedades; y (ii) si dichos cambios evolucionaron hacia la adopción o no adopción de la Norma.

Para la generación de resultados, se clasificaron tipos de respuestas a nivel agregado (Anexo 2) a partir del cumplimiento o incumplimiento de la práctica.

Así, se identificó como Respuesta de Adopción aquellas respuestas que al año 2014 terminan informando su cumplimiento ("Práctica Relevante"). De este total, se determina el subconjunto de prácticas por Sociedades que siempre habrían cumplido con ella ("Práctica Relevante pre-Norma”). En estos dos casos puede afirmarse que las Sociedades consideraron relevantes las prácticas incluso desde antes de su vigencia ${ }^{56}$.

Luego se identificó como Respuesta de No Adopción, las que al año 2014 terminan informando su incumplimiento ("Práctica Irrelevante"). De este total, se determina el subconjunto de prácticas por Sociedades que nunca cumplió con ella (considerándose "Práctica Nunca Relevante"). En estos dos casos puede afirmarse que las Sociedades las consideraron irrelevantes o innecesarias incluso desde antes de su vigencia.

Finalmente, se consideraron los cambios en las respuestas. En primer lugar, se identificó un Cambio a Positivo en las que el 2012 o 2013 fueron negativas, pero terminaron siendo positivas el año 2014 (no-no-sí, no-sí-sí y sí-no-sí). En este caso la práctica se clasificó como "Práctica Relevante" ya que el año 2014 las Sociedades informaron adoptarla aun cuando en alguno de los años anteriores informaron no adoptarla.

En segundo lugar se identificó un Cambio a Negativo en las que en el 2012 o 2013 fueron positivas, pero terminaron siendo negativas el año 2014 (sí-sí-no, sí-no-no y no-síno). En este caso se clasifico como "Práctica Irrelevante", ya que el año 2014 las Sociedades informaron no adoptarla aun cuando en alguno de los años anteriores informaron adoptarla.

\subsection{METOdología de ANÁLISIS CUALITATIVO}

Para la segunda parte se analizó el contenido de las respuestas y explicaciones a la Norma. Es un análisis descriptivo que pretende verificar la calidad de la información divulgada para determinar si la Norma proveyó información relevante a los inversionistas. Para la generación de resultados se evaluó si las respuestas fueron explícitas e informativas,

\footnotetext{
56 Puede argüirse que ciertas Sociedades adoptaron sus estándares antes de la vigencia de la Norma porque se conoció su contenido de manera anticipada. Es decir, las respuestas "relevante pre-Norma" puede subestimar la importancia que tuvo la Norma en cuanto a la adopción de estándares.
} 
y para esto se clasificaron los tipos de respuestas a nivel agregado a partir del contenido y nivel de explicación.

En primer lugar se identificó como Respuesta Estandarizada las que durante los 3 años fueron prácticamente idénticas tomando en cuenta la adopción o no adopción junto con el contenido de la explicación, respondiendo de la misma forma.

En segundo lugar se identificó como Respuesta Formal las que informaron formalmente solo lo indispensable considerando la Norma como una simple exigencia legal.

En tercer lugar se identificó como Respuesta Ambigua aquellas que indicaban cumplir con una práctica pero se explicaba que solo se cumplía parcialmente; o aquellas que para la misma práctica indicaron no cumplir pero explicaban que cumplían con ella en su totalidad. Se busca concluir si la disparidad de explicaciones revelaron distintas concepciones frente a una misma práctica, expresando distintas aproximaciones y respuestas a la misma pregunta considerando que los hechos eran idénticos.

Para verificar la calidad de la información en cuanto a la existencia de Respuestas Formales y Respuestas Ambiguas, se optó por identificar (i) la existencia de cambios en las explicaciones de un año a otro; y (ii) los resultados de estas respuestas al año 2014.

\section{ANÁLISIS CUANTITATIVO DE RESULTADOS}

A continuación se presenta un análisis cuantitativo de los resultados expuestos en cuanto a la variabilidad de cambios en las respuestas.

\subsection{ANÁLISIS CUANTITATIVO SEGÚN SECCIÓN}

Los resultados se presentan en el Cuadro 1. En cuanto al nivel de adopción (Práctica Relevante), se observa que la Sección 4 obtuvo el mayor nivel de adopción, 79\% (aunque las prácticas individuales con el mayor porcentaje de adopción no pertenecían a esta sección). La Sección 2 obtuvo el mayor nivel de no adopción (Práctica Irrelevante), 43,1\%. Sin perjuicio de ello, se debe considerar que una de las prácticas de esta Sección 2 (mecanismos de votación remota) fue durante los 3 años considerada como Práctica Nunca Relevante, lo que incide en el porcentaje total de no adopción de la sección.

En cuanto a la existencia de cambios, la Sección 4 obtuvo el mayor porcentaje de cambios a positivo con 8,8\%. Por el contrario, la Sección 3 obtuvo el mayor porcentaje de cambios a negativo, con $-5,5 \%$. Los cambios a negativo presumiblemente reflejan cierta ambigüedad inicial en las preguntas y que una vez que se tuvo claridad sobre el concepto, se consideró irrelevante.

En términos de proporciones, las prácticas de la Sección 4 fueron consideradas como las más relevantes y las que obtuvieron el mayor porcentaje de cambios a positivo. Con esto se deduce que la Sección 4 sí generó una oportunidad de mejora para las Sociedades. Por otro lado, la Sección 3 fue la sección con mayor proporción de cambios $(12,8 \%)$ tanto a positivo $(7,3 \%)$ como a negativo $(-5,5 \%)$. Esto puede deberse a un problema de redacción e interpretación de las prácticas incluidas en dicha sección. 


\subsection{ANÁLISIS CUANTITATIVO SEGÚN PRÁCTICA}

En cuanto al nivel de adopción (Práctica Relevante), la práctica "Documentación de información” (1.B.c) obtuvo el mayor porcentaje de adopción, 98,8\%. Sin embargo, las respuestas fueron invariables en el tiempo, sin cambios con posterioridad a la emisión de la Norma (Práctica Relevante Pre-Norma). De esto se puede inferir que si bien las Sociedades la considerarían como la práctica más relevante, ella no generó cambios en las Sociedades. La práctica "Página web actualizada" (2.A.f) fue la segunda práctica más adoptada, con $95,1 \%$. Sin embargo las respuestas presentaron un $1,2 \%$ de cambios a positivo pero no en forma significativa, así como un 93,9\% informaron adoptar la práctica durante los tres años (Práctica Relevante Pre-Norma).

En cuanto al nivel de no adopción (Práctica Irrelevante), la práctica "Votación remota en tiempo real" (2.A.b) obtuvo el mayor porcentaje de no adopción, 100\%. Hay que destacar que fue considerada la más irrelevante con anterioridad y con posterioridad a la Norma (Práctica Nunca Relevante). La segunda práctica con mayor porcentaje de no adopción fue "Dedicación mínima mensual directores" (1.B.b), con 93,9\%, respuestas que cambiaron a negativo en $-2,4 \%$ (siendo un porcentaje no significativo). De hecho, un $91,5 \%$ de las Sociedades la consideraron como "Práctica Nunca Relevante."

En cuanto a la existencia de cambios, las respuestas a "Reuniones con las empresas de auditoría externa" (1.A.c) y "Código de conducta ética" (4.A.d) obtuvieron el mayor porcentaje de cambio a positivo, con $15,9 \%$ cada una. Sin embargo para la práctica 1.A.c se observan resultados contradictorios, ya que también obtuvo el mayor porcentaje de cambios a negativo $(-11 \%)$ lo que nuevamente puede explicarse como un problema de ambigüedad en su definición. En todo caso, pareciera que la práctica de tener un código de ética explícito generó una oportunidad de cambio positivo.

Por otro lado, las respuestas "Unidad de relación con inversionistas" (2.A.d) y "Velar por la suficiencia, oportunidad y pertinencia de las revelaciones al mercado" (2.A.e) obtuvieron el segundo mayor porcentaje de cambios a negativo, con un $-9,8 \%$ cada una. Sin embargo también se observa que la segunda de estas obtuvo un mayor porcentaje de cambio a positivo $(8,5 \%)$ a diferencia de la 2. A.d $(3,7 \%)$.

\subsection{INTERPRETACIÓN DE RESULTADOS CUANTITATIVOS}

Es interesante apreciar los resultados agregados considerando todas las prácticas evaluadas. Del total, un 65,3\% sería relevante presentando el mayor nivel de adopción (Práctica Relevante). De dicho porcentaje, 59\% habría sido cumplido previamente a la Norma (Práctica Relevante Pre-Norma), considerando que se respondió afirmativamente desde el principio. Las prácticas no adoptadas son un 34,7\% (Práctica Irrelevante), de las cuales un $30,4 \%$ corresponde a las que nunca fueron adoptadas (Práctica Nunca Relevante).

La proporción de respuestas invariables (más allá de las complicaciones por la ambigüedad en la interpretación de las preguntas) siempre afirmativas (59\%) y siempre negativas $(30,4 \%)$ es $89,4 \%$. Es interesante notar que si tanto las respuestas positivas como negativas son invariables en el tiempo, esto puede reflejar que la Norma no tuvo efectos. Vale decir, el potencial de cambio en las respuestas reportadas por parte de las Sociedades alcanzaría un 10,7\%, pero -como ya se ha mencionado- es posible que algunas prácticas se hayan adoptado anticipándose a la vigencia de la Norma. 
Ahora bien, intentando medir directamente el impacto de la Norma (con las respuestas cambio a positivo) hay un impacto potencial positivo en un $6,4 \%$ de las prácticas. Este número es estadísticamente significativo, pero en términos absolutos sería "pequeño". Las secciones que muestran mayores fracciones de cambio a positivo son la $3^{\text {a }}$ (sustitución y compensación de ejecutivos principales) con 7,3\% y la 4a (definición, implementación y supervisión de políticas y procedimientos de control interno y gestión de riesgos) con $8,8 \%$.

Llama la atención que existan respuestas con un significativo disenso entre empresas que consideran una práctica como necesaria versus otras que no. Las mayores diferencias se encuentran justamente en las respuestas a las secciones 3 y 4 . Esto puede estar reflejando precisamente que "one size doesn't fit all", ya que la mayoría de ellas no cambió su percepción de la importancia de ciertas prácticas. Sin embargo, dado que además son las categorías con mayores cambios hacia respuestas afirmativas, puede estar reflejando cambios reales o al menos formales. No es posible determinar qué fracción de dicho cambio es atribuible a la aversión a riesgos regulatorios.

Las respuestas en que existe un cambio a negativo (habiendo tenido alguna vez una respuesta afirmativa) representan un 4,3\% del total, número que resulta ser estadísticamente significativo, pero en términos absolutos sería "pequeño". Igualmente es difícil pensar que una práctica en algún momento haya sido considerada relevante o importante y luego haya dejado de serlo, suponiendo lo demás constante. La explicación más probable es que el cambio en las respuestas refleje ambigüedad en la formulación de las preguntas.

\section{CUADRO 1 ANÁLISIS DE RESPUESTAS}

El Cuadro 1 resume los resultados del análisis cuantitativo. Cada número representa proporción de "casos favorables" sobre casos totales (frecuencia relativa). Cada resultado va acompañado de asteriscos. Si están presentes, el resultado es estadísticamente significativo y mientras más asteriscos tengan, más significativo resulta ser. Como se ha dicho, la significancia estadística busca determinar la validez del resultado más allá de la muestra. Vale decir, un resultado estadísticamente significativo indicaría que puede generalizarse al universo de empresas similares.

\begin{tabular}{|c|c|c|c|c|c|c|c|c|c|c|c|}
\hline & & \multicolumn{2}{|c|}{$\begin{array}{l}\text { Respuesta } \\
\text { de } \\
\text { Adopción } \\
\text { (Práctica } \\
\text { Relevante) }\end{array}$} & $\begin{array}{l}\text { Práctica } \\
\text { Relevante } \\
\text { Pre-Nor- } \\
\text { ma }\end{array}$ & $\begin{array}{l}\text { Respuesta } \\
\text { de No } \\
\text { Adopción } \\
\text { (Práctica } \\
\text { Irrelevan- } \\
\text { te) }\end{array}$ & \multicolumn{2}{|c|}{$\begin{array}{l}\text { Práctica } \\
\text { Nunca } \\
\text { Relevante }\end{array}$} & \multicolumn{2}{|c|}{$\begin{array}{l}\text { Cambio a } \\
\text { positivo }\end{array}$} & \multicolumn{2}{|c|}{$\begin{array}{l}\text { Cambio a } \\
\text { negativo }\end{array}$} \\
\hline & Sección 1 & 0,622 & ** & $0,556 * *$ & 0,378 ** & 0,334 & ** & 0,066 & & 0,044 & ** \\
\hline 1.A.a) & Proceso Inducción & 0,951 & ** & $0,890 \quad * *$ & $0,049 *$ & 0,049 & * & 0,061 & * & - & - \\
\hline 1.A.b) & $\begin{array}{l}\text { Política contrata- } \\
\text { ción asesores }\end{array}$ & 0,683 & ** & $0,622 * *$ & $0,317 \quad * *$ & 0,293 & ** & 0,061 & $*$ & 0,024 & \\
\hline
\end{tabular}


Godoy Uson, María Paz / Walker Hitschfeld, Eduardo / Zegers Ruiz-TAGle, Matías — Análisis del primer intento...

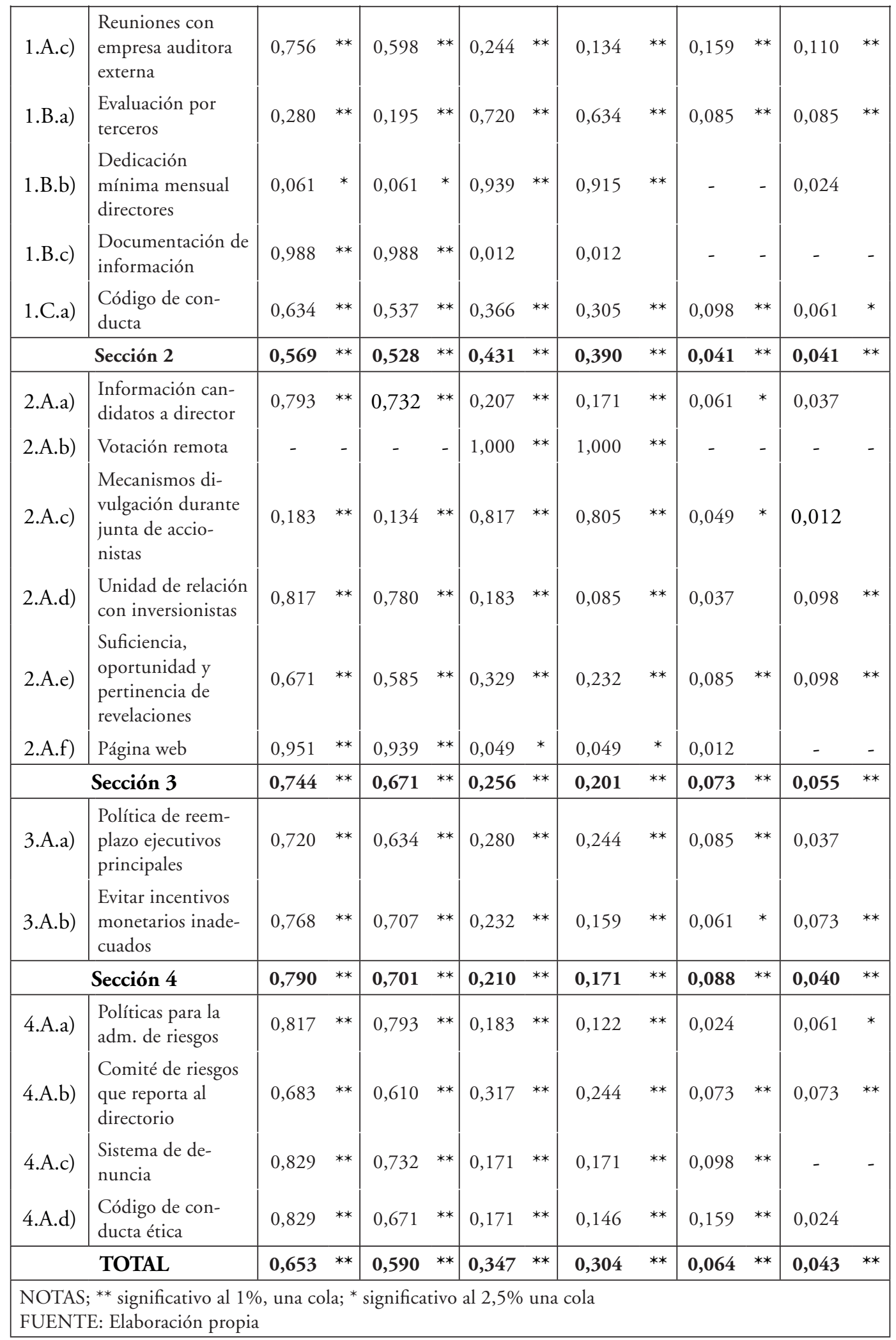




\section{ANÁLISIS CUALITATIVO DE RESULTADOS}

A continuación se presenta un análisis cualitativo de las explicaciones emitidas en cuanto a la calidad de la información. Es un análisis descriptivo que pretende verificar la calidad de la información divulgada para determinar si la Norma logró proveer información relevante al mercado.

\subsection{ANÁLISIS CUALITATIVO SEGÚN PRÁCTICA}

\subsubsection{ESTANDARIZACIÓN DE LAS RESPUESTAS (CUADRO 2)}

En cuanto a las Respuestas Estandarizadas, las prácticas más irrelevantes (según los resultados del análisis cuantitativo) son las más estandarizadas. Así, la Práctica 2.A.b "Votación remota en tiempo real" fue la más estandarizada, con $92,5 \%$ de estandarización. Este resultado coincide con el análisis cuantitativo donde esta práctica fue la menos adoptada, con 100\% (Práctica Nunca Relevante). Asimismo se puede inferir que la diferencia del 7,5\% reflejó una intención de mejorar la explicación aun cuando nunca se adoptó la práctica (la estandarización toma en cuenta tanto las respuestas en cuanto a su adopción/ no adopción como el contenido de la explicación). Este resultado se repite para las dos siguientes prácticas más estandarizadas, 1.B.b "Dedicación mínima mensual directores" con $88,8 \%$, la que al igual que en el caso anterior, fue considerada como la segunda menos adoptada (Práctica Irrelevante en 93,9\% y Práctica Nunca Relevante en 91,5\%); y 2.A.c. "Mecanismos de divulgación oportuna durante la junta de accionistas", con 86,3\% de respuestas estandarizadas.

Por otra parte, las prácticas más adoptadas también obtuvieron altos porcentajes de estandarización (aunque menores en comparación con las menos adoptadas). Así, la 1.B.c "Documentación de información", obtuvo una estandarización de 83,8\%, siendo que esta práctica fue la más adoptada de todas, con 98,8\% (Práctica Relevante y Práctica Relevante Pre-Norma). Un resultado similar ocurre con 1.A.a "Proceso inducción nuevos directores", con 73,8\% de estandarización, y 2.A.f "Página web actualizada" con 82,5\% de estandarización (ambas son las segundas más adoptadas con 95,1\%). Se entiende que la estandarización en las respuestas no debe relacionarse como una variable de medición negativa.

\subsubsection{CAMBIOS EN CALIDAD DE LA INFORMACIÓN: FORMALIDAD Y AMBIGÜEDAD (CUADRO 3)}

En cuanto al análisis de Respuestas Formales y Respuestas Ambiguas estas se evaluaron de dos formas:

(i) Según los cambios que se presentaron de un año a otro:

Se reportaron cambios significativos el 2013, donde la calidad de la información divulgada mejoró producto de la Norma. Esto se reflejó en que las Respuestas Formales y las Respuestas Ambiguas disminuyeron en el periodo 2012-2013, algo que no ocurrió en el periodo 2013-2014. Sin embargo, no es posible determinar que el perfeccionamiento en la calidad de la información haya producido información relevante al mercado ya que en todo momento fue difícil determinar la realidad de las Sociedades.

- Respuestas Formales: 
En total las Respuestas Formales disminuyeron en 2013 un 8,2\% a diferencia del 2014 que solo disminuyeron un $0,6 \%$. Las prácticas con mayores porcentajes de disminución de Respuestas Formales son 3.A.b "Evitar incentivos monetarios inadecuados" $(17,5 \%)$ y 4.A.d "Código de conducta ética" (16,3\%). Para 3.A.b, es difícil interpretar los resultados ya que esta práctica (según los resultados del análisis cuantitativo) no se destacó por presentar porcentajes relevantes de adopción o no adopción, tampoco cambios significativos en este mismo ámbito. Es probable que la SVS haya oficiado a las Sociedades en esta práctica y por lo tanto las respuestas fueron más informativas en el segundo año pero sin generar un cambio en las otras variables. Por el contrario, para 4.A.d. existe una correlación importante entre los resultados del análisis cuantitativo y cualitativo ya que tuvo el mayor cambio a positivo, con 15,9\% (práctica con mayor impacto de la Norma). Es decir, los cambios fueron significativos en ambos análisis ya que las Sociedades la fueron adoptando a lo largo del tiempo y las explicaciones mejoraron notablemente.

- Respuestas Ambiguas:

Las Respuestas Ambiguas disminuyeron en 2013 un 4,3\%, aumentaron levemente un $0,7 \%$ en 2014 . Las prácticas con mayores porcentajes de disminución de Respuestas Ambiguas son 2.A.d "Unidad de relación con inversionistas" con 13,8\% y 1.B.a "Evaluación por tercero", con 10\%. Se puede interpretar que durante 2012 las Sociedades no entendieron la práctica y/o cómo debían responderla (de lo que a su vez se infiere que las prácticas no estarían bien redactadas). Así, en 2.A.d, existió una evidente confusión con respecto a la persona o unidad encargada de informar a los inversionistas sobre la situación y marcha de los negocios. Esta disminución significó que las Sociedades entendieron mejor la práctica desde 2013 lo que permitió mejorar la calidad de la información. Se destaca también que esta misma práctica se identificó como una con más cambios a negativo, un $9,8 \%$, por lo que se puede deducir que las Sociedades al entenderla terminaron por no adoptarla.

(ii) Según el nivel de formalidad y ambigüedad en 2014:

Según los resultados del 2014, las respuestas más formales fueron 2.A.a "Información, experiencia y perfil de candidatos a director", con 51,3\%, mientras que las respuestas más ambiguas fueron en la 1.A.c "Reuniones con empresas auditoras externas", con 26,3\%.

En particular, para 2.A.a se puede interpretar que las Sociedades consideraron innecesario entregar información adicional ya que en parte se encuentra regulada en la ley. Asimismo, la 1.A.c tal como se mencionó en el análisis cuantitativo generó los resultados más contradictorios puesto que generó mayor cambio a positivo $(15,9 \%)$ y mayor cambio a negativo $(-11 \%)$. Se observa que en 2014 las respuestas para esta práctica fueron las más ambiguas $(26,3 \%)$ y unas de las más formales (45\%). Estos resultados permiten deducir que la práctica estaría mal planteada ya que dentro de ella se requerían un conjunto de requisitos difícilmente alcanzables, con lo que las Sociedades no entendieron cómo responderla.

\subsection{INTERPRETACIÓN DE RESULTADOS CUALITATIVOS.}

(iii) Estandarización (respuestas idénticas durante los tres años):

Se observa un gran número de respuestas estandarizadas. Un 65,8\% de las Sociedades no variaron sus explicaciones, e incluso algunas se limitaron a informar que no exis- 
tieron cambios remitiéndose a las respuestas del año anterior (respuestas 2015 respecto a 2014).

Formalidad (cumplimiento formal de la norma)

Las Respuestas Formales disminuyeron en forma importante el 2013 (8,2\%), reconociéndose una mejora en la calidad de la información a partir de la Norma. Sin embargo, al ser la Norma similar a un cuestionario, la información resultó ser homogénea y con poco nivel de explicaciones que entreguen más información real sobre la forma en que las Sociedades han implementado la práctica. Según los datos recogidos en las explicaciones, las Sociedades informaron solo lo indispensable considerando la Norma como una exigencia legal. A pesar de reportarse una mejora el 2013, la información no fue concluyente para determinar de qué forma estaban adoptando la práctica o porque no lo hacían. Más aún, el 2014 las Respuestas Formales fueron un 39,3\%. Se puede deducir una aversión generalizada al riesgo por incumplimiento a partir de la escasa información divulgada por respuesta, particularmente el 2012. Es decir las Sociedades habrían sido cautelosas al responder, evitando someterse a posibles sanciones por parte del regulador en caso de existir errores $\mathrm{u}$ omisiones. A pesar de la cuantificación de datos y calificación de la información, es difícil establecer en este análisis si los porcentajes en niveles de adopción reflejan la realidad corporativa o si solo están divulgando lo mínimo para cumplir formalmente con la regulación. No hay que olvidar que el emisor de la Norma es el regulador por tanto puede esperarse que el tipo de cumplimiento sea solo formal y no cualitativo.

(iv) Ambigüedad (disparidad de criterios en las respuestas):

Las Respuestas Ambiguas también disminuyeron el 2013 (4,3\%), sin embargo (a diferencia de la disminución de Respuestas Formales), este número no fue significativo. Se observó que dentro de una misma práctica, las explicaciones emitidas por las Sociedades tendieron a ser variadas. Si bien se espera que la explicación de no adopción sea distinta entre las Sociedades, lo cierto es que muchas veces la disparidad de explicaciones reveló distintas concepciones frente a una misma práctica, expresando distintas aproximaciones y respuestas siendo que los hechos eran idénticos. Esto quedó reflejado al constatarse por ejemplo que se indicaba cumplir con una práctica pero al mismo tiempo se explicaba que solo se cumplía parcialmente. El 2014 las Respuestas Ambiguas fueron un 12\%. Es muy difícil dar a conocer la realidad en cuanto a la adopción de estándares si la misma normativa sugiere divulgar un doble comportamiento, es decir; cumplo con la práctica, pero no cumplo según la respuesta; o al revés no cumplo con la práctica, pero sí cumplo según la respuesta.

Cuando la Norma fue emitida, la SVS no dio ninguna orientación formal sobre Principios de GC, ni emitió algún documento que explicara los principios que inspiraban las prácticas, lo que llevó a que cada Sociedad la interpretara de forma distinta. Esta falta de guía se vio subsanada en parte por los Informes emitidos por la SVS en la puesta en consulta de la NCG 385, ya que por primera vez la SVS intentó explicar el alcance de las prácticas propuestas, lo que provocaría una mejor aproximación de las Sociedades a dicha norma. Sin embargo en la NCG 385 hay una notoria ausencia de vínculo entre el antecedente, la práctica y la realidad en Chile sin que se indique de qué forma cada antecedente presentado ha influido en la práctica respectiva. 


\section{CUADRO 2 ANÁLISIS CUALITATIVO DE RESPUESTAS ESTANDARIZACIÓN}

Este resume los resultados del análisis cualitativo respecto a la estandarización en las respuestas. Cada número representa proporción de "casos favorables" sobre casos totales (frecuencia relativa). Cada resultado va acompañado de asteriscos. Si están presentes, el resultado es estadísticamente significativo y mientras más asteriscos tenga, más significativo resulta ser. Como se ha dicho, la significancia estadística busca determinar la validez del resultado más allá de la muestra.

\begin{tabular}{|c|c|c|c|}
\hline \multicolumn{4}{|c|}{ Estandarización } \\
\hline \multicolumn{2}{|r|}{ Sección 1} & $74,2 \%$ & ** \\
\hline 1.A.a) & Proceso Inducción & $73,8 \%$ & $* *$ \\
\hline 1.A.b) & Política contratación asesores & $78,3 \%$ & ** \\
\hline 1.A.c) & Reuniones con empresa auditora externa & $62,5 \%$ & ** \\
\hline 1.B.a) & Evaluación por terceros & $80,0 \%$ & $* *$ \\
\hline 1.B.b) & Dedicación mínima mensual directores & $88,8 \%$ & $* *$ \\
\hline 1.B.c) & Documentación de información & $83,8 \%$ & ** \\
\hline 1.C.a) & Código de conducta & $52,5 \%$ & $* *$ \\
\hline \multicolumn{2}{|r|}{ Sección 2} & $77,9 \%$ & ** \\
\hline 2.A.a) & Información de candidatos a director & $67,5 \%$ & $* *$ \\
\hline 2.A.b) & Votación remota & $92,5 \%$ & $* *$ \\
\hline 2.A.c) & $\begin{array}{l}\text { Mecanismos divulgación durante junta } \\
\text { de accionistas }\end{array}$ & $86,3 \%$ & $* *$ \\
\hline 2.A.d) & Unidad de relación con inversionistas & $75,0 \%$ & ** \\
\hline 2.A.e) & $\begin{array}{c}\text { Suficiencia, oportunidad y pertinencia de } \\
\text { revelaciones }\end{array}$ & $63,8 \%$ & $* *$ \\
\hline 2.A.f) & Página web & $82,5 \%$ & $* *$ \\
\hline \multicolumn{2}{|r|}{ Sección 3} & $56,3 \%$ & $* *$ \\
\hline 3.A.a) & $\begin{array}{c}\text { Política de reemplazo ejecutivos } \\
\text { principales }\end{array}$ & $60,0 \%$ & $* *$ \\
\hline 3.A.b) & Evitar incentivos monetarios inadecuados & $52,5 \%$ & $* *$ \\
\hline \multicolumn{2}{|r|}{ Sección 4} & $55,0 \%$ & ** \\
\hline 4.A.a) & Políticas adm. de riesgos & $42,5 \%$ & $* *$ \\
\hline 4.A.b) & $\begin{array}{l}\text { Comité de riesgos que reporta al } \\
\text { directorio }\end{array}$ & $66,3 \%$ & $* *$ \\
\hline 4.A.c) & Sistema de denuncia & $68,8 \%$ & $* *$ \\
\hline 4.A.d) & Código de conducta ética & $42,5 \%$ & $* *$ \\
\hline \multicolumn{2}{|r|}{ TOTAL } & $65,8 \%$ & $* *$ \\
\hline
\end{tabular}




\section{CUADRO 3 \\ ANÁLISIS CUALITATIVO DE RESPUESTAS FORMALIDAD - AMBIGÜEDAD}

Este resume los resultados del análisis cualitativo respecto a la formalidad y ambigüedad en las respuestas. Se analizó la variación anual. Cada resultado va acompañado de asteriscos. Si están presentes, el resultado es estadísticamente significativo y mientras más asteriscos tenga, más significativo resulta ser. Como se ha dicho, la significancia estadística busca determinar la validez del resultado más allá de la muestra.

\begin{tabular}{|c|c|c|c|c|c|c|c|c|c|c|c|}
\hline & & \multicolumn{2}{|c|}{2014} & \multicolumn{4}{|c|}{ Cambio 2012 a 2013} & \multicolumn{4}{|c|}{ Cambio 2013 a 2014} \\
\hline & & $\begin{array}{l}\text { Forma- } \\
\text { lidad }\end{array}$ & $\begin{array}{l}\text { Ambi- } \\
\text { güedad }\end{array}$ & $\begin{array}{c}\text { Formali- } \\
\text { dad }\end{array}$ & $\begin{array}{l}\text { Signifi- } \\
\text { cancia }\end{array}$ & $\begin{array}{l}\text { Ambi- } \\
\text { güedad }\end{array}$ & $\begin{array}{l}\text { Signifi- } \\
\text { cancia }\end{array}$ & $\begin{array}{c}\text { Formali- } \\
\text { dad }\end{array}$ & $\begin{array}{l}\text { Signifi- } \\
\text { cancia }\end{array}$ & $\begin{array}{l}\text { Ambi- } \\
\text { güedad }\end{array}$ & $\begin{array}{l}\text { Signifi- } \\
\text { cancia }\end{array}$ \\
\hline & Sección 1 & $40,0 \%$ & $13,6 \%$ & $-4,7 \%$ & * & $-4,5 \%$ & & $-0,1 \%$ & & $0,4 \%$ & \\
\hline 1.A.a) & $\begin{array}{c}\text { Proceso } \\
\text { Inducción }\end{array}$ & $36,3 \%$ & $7,5 \%$ & $-3,8 \%$ & & $-1,3 \%$ & & $0,0 \%$ & & $0,0 \%$ & \\
\hline 1.A.b) & \begin{tabular}{|c|} 
Política \\
contratación \\
asesores
\end{tabular} & $47,5 \%$ & $21,3 \%$ & $-4,2 \%$ & & $-3,8 \%$ & & $0,8 \%$ & & $0,0 \%$ & \\
\hline 1.A.c) & \begin{tabular}{|c|} 
Reuniones \\
con empresa \\
auditora \\
externa
\end{tabular} & $45,0 \%$ & $26,3 \%$ & $-5,0 \%$ & * & $-8,8 \%$ & $* *$ & $2,5 \%$ & & $0,0 \%$ & \\
\hline 1.B.a) & $\begin{array}{l}\text { Evaluación } \\
\text { por terceros }\end{array}$ & $38,8 \%$ & $16,3 \%$ & $-1,3 \%$ & & $-10,0 \%$ & $* *$ & $0,0 \%$ & & $1,3 \%$ & \\
\hline 1.B.b) & $\begin{array}{l}\text { Dedicación } \\
\text { mínima } \\
\text { mensual } \\
\text { directores }\end{array}$ & $26,3 \%$ & $6,3 \%$ & $-1,3 \%$ & & $-5,0 \%$ & * & $-1,3 \%$ & & $0,0 \%$ & \\
\hline 1.B.c) & $\begin{array}{c}\text { Documen- } \\
\text { tación de } \\
\text { información }\end{array}$ & $43,8 \%$ & $11,3 \%$ & $-5,0 \%$ & * & $-2,5 \%$ & & $0,0 \%$ & & $0,0 \%$ & \\
\hline 1.C.a) & $\begin{array}{l}\text { Código de } \\
\text { conducta }\end{array}$ & $42,5 \%$ & $6,3 \%$ & $-12,5 \%$ & $* *$ & $0,0 \%$ & - & $-2,5 \%$ & & $1,3 \%$ & \\
\hline \multicolumn{2}{|c|}{ Sección 2} & $35,6 \%$ & $9,6 \%$ & $-3,5 \%$ & & $-4,4 \%$ & & $-1,3 \%$ & & $0,4 \%$ & \\
\hline 2.A.a) & \begin{tabular}{|c|} 
Información \\
candidatos a \\
director
\end{tabular} & $51,3 \%$ & $8,8 \%$ & $-5,0 \%$ & $*$ & $-3,8 \%$ & & $-3,8 \%$ & & $2,5 \%$ & \\
\hline 2.A.b) & $\begin{array}{c}\text { Votación } \\
\text { remota }\end{array}$ & $36,3 \%$ & $2,5 \%$ & $-2,5 \%$ & & $-1,3 \%$ & & $0,0 \%$ & & $0,0 \%$ & \\
\hline 2.A.c) & \begin{tabular}{|c|} 
Mecanismos \\
divulgación \\
durante junta \\
de accionistas
\end{tabular} & $37,5 \%$ & $7,5 \%$ & $-2,5 \%$ & & $-1,3 \%$ & & $0,0 \%$ & & $0,0 \%$ & \\
\hline
\end{tabular}




\begin{tabular}{|c|c|c|c|c|c|c|c|c|c|}
\hline 2.A.d) & $\begin{array}{l}\text { Unidad de } \\
\text { relación con } \\
\text { inversionistas }\end{array}$ & $43,8 \%$ & $22,5 \%$ & $2,5 \%$ & & $-13,8 \%$ & $* *$ & $-3,8 \%$ & $0,0 \%$ \\
\hline 2.A.e) & $\begin{array}{c}\text { Suficiencia, } \\
\text { oportunidad } \\
\text { y pertinencia } \\
\text { de revelacio- } \\
\text { nes }\end{array}$ & $37,5 \%$ & $15,0 \%$ & $-8,8 \%$ & $* *$ & $-5,0 \%$ & $*$ & $0,0 \%$ & $0,0 \%$ \\
\hline 2.A.f) & Página web & $7,5 \%$ & $1,3 \%$ & $-5,0 \%$ & $*$ & $-1,3 \%$ & & $0,0 \%$ & $0,0 \%$ \\
\hline \multicolumn{2}{|c|}{ Sección 3} & $43,1 \%$ & $14,4 \%$ & $-15,0 \%$ & $* *$ & $-5,0 \%$ & $*$ & $0,0 \%$ & $1,3 \%$ \\
\hline 3.A.a) & $\begin{array}{l}\text { Política de } \\
\text { reemplazo } \\
\text { ejecutivos } \\
\text { principales }\end{array}$ & $41,3 \%$ & $11,3 \%$ & $-12,5 \%$ & $* *$ & $-3,8 \%$ & & $0,0 \%$ & $1,3 \%$ \\
\hline 3.A.b) & $\begin{array}{c}\text { Evitar } \\
\text { incentivos } \\
\text { monetarios } \\
\text { inadecuados }\end{array}$ & $45,0 \%$ & $17,5 \%$ & $-17,5 \%$ & $* *$ & $-6,3 \%$ & $*$ & $0,0 \%$ & $1,3 \%$ \\
\hline \multicolumn{2}{|c|}{ Sección 4} & $38,4 \%$ & $10,3 \%$ & $-9,4 \%$ & $* *$ & $-3,4 \%$ & & $-0,9 \%$ & $0,6 \%$ \\
\hline 4.A.a) & $\begin{array}{l}\text { Políticas } \\
\text { adm. de } \\
\text { riesgos }\end{array}$ & $33,8 \%$ & $10,0 \%$ & $-13,8 \%$ & $* *$ & $-2,5 \%$ & & $-1,3 \%$ & $0,0 \%$ \\
\hline 4.A.b) & $\begin{array}{c}\text { Comité de } \\
\text { riesgos que } \\
\text { reporta al } \\
\text { directorio }\end{array}$ & $43,8 \%$ & $11,3 \%$ & $-2,5 \%$ & & $-1,3 \%$ & & $-2,5 \%$ & $0,0 \%$ \\
\hline 4.A.c) & $\begin{array}{l}\text { Sistema de } \\
\text { denuncia }\end{array}$ & $36,3 \%$ & $7,5 \%$ & $-5,0 \%$ & * & $-1,3 \%$ & & $0,0 \%$ & $1,3 \%$ \\
\hline 4.A.d) & $\begin{array}{l}\text { Código de } \\
\text { conducta } \\
\text { ética }\end{array}$ & $40,0 \%$ & $12,5 \%$ & $-16,3 \%$ & $* *$ & $-8,8 \%$ & ** & $0,0 \%$ & $1,3 \%$ \\
\hline \multicolumn{2}{|r|}{ TOTAL } & $39,3 \%$ & $12,0 \%$ & $-8,2 \%$ & $* *$ & $-4,3 \%$ & & $-0,6 \%$ & $0,7 \%$ \\
\hline
\end{tabular}

\subsection{OTRAS PRÁCTICAS}

Un análisis particular merece la Sección 5, referida a otras Prácticas de GC distintas a las propuestas por la SVS. Al respecto, un 29\% de las Sociedades entregaron información aunque poco o nada variaron durante su vigencia.

Sin perjuicio de esto, cabe destacar, entre otras, las siguientes prácticas:

- $\quad$ El énfasis puesto en el aporte a la comunidad (Responsabilidad Social Corporativa o Políticas referidas al medio ambiente).

- $\quad$ Existencia de Códigos o Manuales de Gobierno Corporativo.

- Modelos de buenas prácticas, como el de prevención del delito o de cumplimiento de libre competencia.

- Existencia de Manual de Gestión Integrado, con distintos procedimientos y certificados.

- Existencia de Gerente de Cumplimiento para velar por las normativas sociales.

- Acceso directo a la información de la administración de la Sociedad para los directores. 
En relación a las respuestas entregadas por las empresas para los tres años de respuesta de la Norma, de las 97 sociedades IGPA consideradas, solo 25 sociedades hicieron mención a la realización de otras prácticas el 2012, 26 el 2013 (incluyendo las del año anterior) y 36 el 2014. Existen 21 sociedades que informaron presentar otras prácticas los tres años del estudio pero sin variación alguna.

\section{CONCLUSIONES}

La NCG 341 es el primer intento de exigir que se haga explícito algún grado de autorregulación referente a estándares de gobierno corporativo en Chile. Que el regulador imponga la obligación de informar bajo un formato de "autorregulación" es un oxímoron, ya que supone una contradicción en sí misma. Considerando que la Norma se ha reemplazado por otra más ambiciosa y detallada -NCG 385- resulta interesante analizar las respuestas dadas a la NCG 341, lo que serviría de base para el perfeccionamiento de la normativa que busca mejorar los estándares de Prácticas de GC en Chile.

La heterogeneidad en algunas respuestas indicaría que las Sociedades cumplieron con la Norma solo cuando esta se ajustaba a sus realidades y circunstancias aplicándose por tanto el principio "one size does not fit all'. Así por ejemplo se observó en las secciones 3 (De la sustitución y compensación de ejecutivos principales) y 4 (de la definición, implementación y supervisión de políticas y procedimientos de control interno y gestión de riesgos en la empresa) en que existió una significativa divergencia entre Sociedades que consideraron una práctica como necesaria versus otras que no, y en ello fueron consistentes ya que no cambiaron su respuesta durante los años de aplicación de la Norma. El llamado a la flexibilidad y autonomía para cumplir con los estándares resulta evidente.

En general, la medición del impacto de la Norma confirmó la existencia de algunos cambios en las respuestas, aunque en términos absolutos no representa un número importante, ya que solo un 10,7\% de las Sociedades reportaron cambios. Por lo tanto, la eficacia del proceso es debatible. Asimismo, el 59\% de las Sociedades que sí adoptaron ciertas prácticas desde el principio bien pudieron anticiparse a su vigencia mientras estaba en consulta o incluso corresponder a prácticas ya existentes, lo que acrecienta las dudas sobre su eficacia.

El análisis confirmó la existencia un impacto potencial positivo en un 6,4\% y negativo en un $4,3 \%$ de las prácticas, números que resultan ser significativos estadísticamente. Es decir, por un lado ciertas Sociedades consideraron algunos aspectos de la Norma como una oportunidad de adoptar prácticas presumiblemente recomendables, al incorporar prácticas que no tenían en un comienzo. Por otro lado, la Norma también generó un efecto contrario, al verificarse que algunas Sociedades reportaron cumplir con ciertas prácticas en un inicio y luego dejaron de hacerlo. Este último porcentaje podría bien explicarse a partir de la ambigüedad en la formulación de las preguntas contenidas en la Norma.

En particular, los resultados arrojaron que a partir de la Norma se generaron cambios significativos en las respuestas de 4 prácticas. Las prácticas con mayores cambios a positivo fueron "Código de conducta ética" y "Reuniones con la empresa auditora externa", cada una con un incremento reportado de $15,9 \%$. Sin embargo con respecto a esta última práctica se reportaron resultados contradictorios, ya que al mismo tiempo obtuvo el mayor porcentaje de cambios a negativo, con un $-11 \%$, lo que puede deberse a un problema de 
ambigüedad en su definición. Otras prácticas con mayores cambios a negativo fueron "Unidad de relación con inversionistas" y "Velar por la suficiencia, oportunidad y pertinencia de las revelaciones al mercado", ambas con un $9,8 \%$.

Por otro lado, hay que destacar que las Sociedades no presentaron cambios significativos en tres las respuestas ("Documentación de información", "Votación remota en tiempo real" y "Página web actualizada").

En general se concluye que los cambios no fueron materiales, por lo que la Norma en sí misma no necesariamente implicó avances en cuanto a Prácticas de GC, tal como era la intención declarada del regulador.

En cuanto a la calidad de la información, si bien se observó un mejoramiento, especialmente el 2013, en general la Norma no proveería información relevante al mercado respecto de las Prácticas de GC adoptadas por las Sociedades, posiblemente porque informaron por decisión de la autoridad y no necesariamente por convicción. Esto puede reflejar aversión de riesgo por incumplimiento, y un ejemplo es que las explicaciones de no adopción no contribuyen en la identificación de problemas y necesidades reales. La evidente estandarización en la secuencia anual de respuestas entrega un tipo de información deficiente lo que hace difícil interpretarlas. En promedio, durante los tres años un 65,8\% de las Sociedades emitieron Respuestas Estandarizadas (es decir, sin cambio en la respuesta).

Además, un 29\% de las Sociedades informaron otras prácticas no enunciadas en la Norma. Sin embargo, no se logró obtener información concluyente con respecto a la relevancia y el nivel real de adopción por las Sociedades de ellas. En este sentido, las Sociedades habrían sido renuentes a entregar más información de la requerida en la Norma.

Es complejo determinar los casos en que hubo cambios materiales en los estándares de Gobierno Corporativo (o al menos, en pocos casos ello fue informado a través de la respuesta de la Norma). Si bien es cierto que no puede concluirse que no ha habido evolución en las Prácticas de GC, lo cierto es que las Sociedades no reportaron a través de la Norma cambios mayores ni tampoco evidenciaron una evolución anual en los fundamentos que permita concluir que esta evolución si existió. Las explicaciones a esa evidencia pueden ser diversas, desde que la entidad podría tener incentivos naturales a limitar la entrega de mayor información por el riesgo de cumplimiento, o las responsabilidades adicionales inherentes a la difusión de información, tanto desde la perspectiva del regulador como de sus accionistas, hasta otras menos triviales, que podrían vincularse con la inexperiencia, desinformación o desconocimiento que podrían tener los directores de esas entidades sobre los beneficios asociados a la difusión de información sobre las Prácticas de GC adoptadas por las Sociedades.

Por último, a partir de los resultados encontrados, es posible plantear una hipótesis fundada sobre el potencial impacto que tendrá la NCG 385. En efecto, en lo referido a Prácticas de GC, fruto de la NCG 341 se reportaron cambios poco relevantes y, con frecuencia, respuestas meramente formales. Esto ocurrió a pesar de que la Norma se refería a un conjunto acotado de prácticas. La NCG 385 evalúa un profuso número de prácticas, que en buena medida son el resultado de la desagregación de las prácticas contenidas en la Norma anterior. Por lo tanto, no sería esperable que la nueva norma tenga un impacto significativo sobre las Prácticas de GC en las Sociedades. 


\section{ANEXO 1}

Muestra de 97 sociedades del IGPA durante los años 2012-2014. Del total de respuestas de las Sociedades se analizaron solo aquellas que se encontraban disponibles en la página web de la SVS al 25/9/15, descartando 15 de ellas.

\begin{tabular}{|c|c|c|c|c|c|c|c|c|c|c|c|c|c|c|}
\hline & Empresas IGPA & 2012 & 2013 & 2014 & & $\begin{array}{c}\text { Empresas } \\
\text { IGPA }\end{array}$ & 2012 & 2013 & 2014 & & $\begin{array}{c}\text { Empresas } \\
\text { IGPA }\end{array}$ & 2012 & 2013 & 2014 \\
\hline 1 & AFP Cuprum & $\mathrm{P}$ & $\mathrm{P}$ & $\mathrm{O}$ & 36 & $\begin{array}{l}\text { Emb. Andina } \\
\text { Serie A }\end{array}$ & $\mathrm{P}$ & $\mathrm{P}$ & $\mathrm{P}$ & 71 & $\begin{array}{l}\text { Molibdenos y } \\
\text { Metales }\end{array}$ & $\mathrm{P}$ & $\mathrm{P}$ & $\mathrm{P}$ \\
\hline 2 & AFP Habitat & $\mathrm{P}$ & $\mathrm{P}$ & $\mathrm{P}$ & 37 & $\begin{array}{l}\text { Emp. Elec. } \\
\text { Pehuenche }\end{array}$ & $\mathrm{P}$ & $\mathrm{P}$ & $\mathrm{P}$ & 72 & $\begin{array}{l}\text { Multiexport } \\
\text { Foods }\end{array}$ & $\mathrm{P}$ & $\mathrm{P}$ & $\mathrm{P}$ \\
\hline 3 & AFP Provida & $\mathrm{P}$ & $\mathrm{P}$ & $\mathrm{P}$ & 38 & $\begin{array}{l}\text { Emp. Elec. } \\
\text { Pilmaiquén }\end{array}$ & $\mathrm{P}$ & $\mathrm{P}$ & $\mathrm{P}$ & 73 & Norte Grande & $\mathrm{P}$ & $\mathrm{P}$ & $\mathrm{P}$ \\
\hline 4 & AES Gener & $\mathrm{P}$ & $\mathrm{P}$ & $\mathrm{P}$ & 39 & $\begin{array}{l}\text { Emp. Nac. de } \\
\text { Electricidad }\end{array}$ & $\mathrm{P}$ & $\mathrm{P}$ & $\mathrm{P}$ & 74 & Parque Arauco & $\mathrm{P}$ & $\mathrm{P}$ & $\mathrm{P}$ \\
\hline 5 & Aguas Andina & $\mathrm{P}$ & $\mathrm{P}$ & $\mathrm{P}$ & 40 & $\begin{array}{l}\text { Emp. Nac. de } \\
\text { Telecomuni- } \\
\text { caciones }\end{array}$ & $\mathrm{P}$ & $\mathrm{O}$ & $\mathrm{P}$ & 75 & Paz Corp & $\mathrm{P}$ & $\mathrm{P}$ & $\mathrm{P}$ \\
\hline 6 & Almendral & $\mathrm{P}$ & $\mathrm{P}$ & $\mathrm{P}$ & 41 & $\begin{array}{l}\text { Emp. Aqua- } \\
\text { chile }\end{array}$ & $\mathrm{P}$ & $\mathrm{P}$ & $\mathrm{P}$ & 76 & $\begin{array}{l}\text { Potasios de } \\
\text { Chile }\end{array}$ & $\mathrm{P}$ & $\mathrm{P}$ & $\mathrm{P}$ \\
\hline 7 & Antarchile & $\mathrm{P}$ & $\mathrm{P}$ & $\mathrm{P}$ & 42 & Emp. CMPC & $\mathrm{P}$ & $\mathrm{P}$ & $\mathrm{P}$ & 77 & Puerto Ventanas & $\mathrm{P}$ & $\mathrm{P}$ & $\mathrm{P}$ \\
\hline 8 & Australis Seafoods & $\mathrm{P}$ & $\mathrm{P}$ & $\mathrm{P}$ & 43 & $\begin{array}{l}\text { Emp. Const. } \\
\text { Moller\&Pérez } \\
\text { Cotapos }\end{array}$ & $\mathrm{O}$ & $\mathrm{P}$ & $\mathrm{P}$ & 78 & Quiñenco & $\mathrm{P}$ & $\mathrm{P}$ & $\mathrm{P}$ \\
\hline 9 & Azul Azul & $\mathrm{P}$ & $\mathrm{O}$ & $\mathrm{P}$ & 44 & Emp. Copec & $\mathrm{P}$ & $\mathrm{P}$ & $\mathrm{P}$ & 79 & Ripley Corp. & $\mathrm{P}$ & $\mathrm{P}$ & $\mathrm{P}$ \\
\hline 10 & Banmédica & $\mathrm{P}$ & $\mathrm{P}$ & $\mathrm{P}$ & 45 & Emp. Hites & $\mathrm{P}$ & $\mathrm{P}$ & $\mathrm{P}$ & 80 & $\begin{array}{l}\text { S.A.C.I. Fala- } \\
\text { bella }\end{array}$ & $\mathrm{P}$ & $\mathrm{P}$ & $\mathrm{P}$ \\
\hline 11 & Banvida & $\mathrm{P}$ & $\mathrm{P}$ & $\mathrm{P}$ & 46 & Emp. Iansa & $\mathrm{P}$ & $\mathrm{P}$ & $\mathrm{P}$ & 81 & Salfacorp & $\mathrm{P}$ & $\mathrm{P}$ & $\mathrm{P}$ \\
\hline 12 & Besalco & $\mathrm{P}$ & $\mathrm{P}$ & $\mathrm{P}$ & 47 & Emp. La Polar & $\mathrm{P}$ & $\mathrm{P}$ & $\mathrm{P}$ & 82 & \begin{tabular}{|l} 
Schwager \\
Energy
\end{tabular} & $\mathrm{P}$ & $\mathrm{P}$ & $\mathrm{O}$ \\
\hline 13 & Blanco y Negro & $\mathrm{P}$ & $\mathrm{P}$ & $\mathrm{O}$ & 48 & $\begin{array}{l}\text { Emp. Tatter- } \\
\text { sall }\end{array}$ & $\mathrm{P}$ & $\mathrm{P}$ & $\mathrm{O}$ & 83 & Sigdo Koppers & $\mathrm{P}$ & $\mathrm{P}$ & $\mathrm{P}$ \\
\hline 14 & Blumar & $\mathrm{O}$ & $\mathrm{P}$ & $\mathrm{P}$ & 49 & Enaex & $\mathrm{P}$ & $\mathrm{P}$ & $\mathrm{P}$ & 84 & $\begin{array}{l}\text { Soc. de Inv. } \\
\text { Campos Chi- } \\
\text { lenos }\end{array}$ & $\mathrm{P}$ & $\mathrm{P}$ & $\mathrm{P}$ \\
\hline 15 & CAP & $\mathrm{P}$ & $\mathrm{P}$ & $\mathrm{P}$ & 50 & Enersis & $\mathrm{P}$ & $\mathrm{P}$ & $\mathrm{P}$ & 85 & $\begin{array}{l}\text { Soc. de Inv. Oro } \\
\text { Blanco }\end{array}$ & $\mathrm{P}$ & $\mathrm{P}$ & $\mathrm{P}$ \\
\hline 16 & $\begin{array}{l}\text { Cemento Pol- } \\
\text { paico }\end{array}$ & $\mathrm{P}$ & $\mathrm{P}$ & $\mathrm{O}$ & 51 & Enjoy & $\mathrm{P}$ & $\mathrm{P}$ & $\mathrm{P}$ & 86 & $\begin{array}{l}\text { Soc. Matriz } \\
\text { SAAM }\end{array}$ & $\mathrm{P}$ & $\mathrm{P}$ & $\mathrm{P}$ \\
\hline 17 & Cemento Bío Bío & $\mathrm{P}$ & $\mathrm{P}$ & $\mathrm{P}$ & 52 & $\begin{array}{l}\text { Env. del } \\
\text { Pacífico }\end{array}$ & $\mathrm{P}$ & $\mathrm{P}$ & $\mathrm{P}$ & 87 & $\begin{array}{l}\text { Soc. Punta del } \\
\text { Cobre Serie A }\end{array}$ & $\mathrm{P}$ & $\mathrm{P}$ & $\mathrm{P}$ \\
\hline 18 & Cencosud & $\mathrm{P}$ & $\mathrm{P}$ & $\mathrm{P}$ & 53 & $\begin{array}{l}\text { Forestal } \\
\text { Const. } \\
\text { Comercial } \\
\text { Pacífico Sur }\end{array}$ & $\mathrm{P}$ & $\mathrm{P}$ & $\mathrm{P}$ & 88 & $\begin{array}{l}\text { Soc. Química y } \\
\text { Minera de Chile } \\
\text { Serie A }\end{array}$ & $\mathrm{P}$ & $\mathrm{P}$ & $\mathrm{P}$ \\
\hline
\end{tabular}




\begin{tabular}{|c|c|c|c|c|c|c|c|c|c|c|c|c|c|c|}
\hline 19 & $\begin{array}{l}\text { CFR Pharmaceu- } \\
\text { ticals }\end{array}$ & $\mathrm{P}$ & $\mathrm{P}$ & $\mathrm{O}$ & 54 & Forus & $\mathrm{P}$ & P & $\mathrm{P}$ & 89 & Socovesa & $\mathrm{P}$ & $\mathrm{P}$ & $\mathrm{P}$ \\
\hline 20 & Cintac & $\mathrm{P}$ & $\mathrm{P}$ & $\mathrm{P}$ & 55 & Gasco & P & P & $\mathrm{P}$ & 90 & Sonda & $\mathrm{P}$ & $\mathrm{P}$ & $\mathrm{P}$ \\
\hline 21 & $\begin{array}{l}\text { Clínica Las } \\
\text { Condes }\end{array}$ & $\mathrm{P}$ & $\mathrm{P}$ & $\mathrm{P}$ & 56 & $\begin{array}{l}\text { Grupo Secu- } \\
\text { rity }\end{array}$ & $\mathrm{P}$ & P & $\mathrm{P}$ & 91 & $\begin{array}{l}\text { Soquimich } \\
\text { Comercial }\end{array}$ & $\mathrm{P}$ & $\mathrm{P}$ & $\mathrm{P}$ \\
\hline 22 & $\begin{array}{l}\text { Coca Cola Em- } \\
\text { bonor Serie A }\end{array}$ & $\mathrm{P}$ & $\mathrm{P}$ & $\mathrm{P}$ & 57 & Hortifrut & $\mathrm{P}$ & P & $\mathrm{P}$ & 92 & Teckpack & $\mathrm{O}$ & $\mathrm{O}$ & $\mathrm{P}$ \\
\hline 23 & Colbún & $\mathrm{P}$ & $\mathrm{P}$ & $\mathrm{P}$ & 58 & Ingevec & $\mathrm{P}$ & $\mathrm{P}$ & $\mathrm{P}$ & 93 & $\begin{array}{l}\text { Viña Concha y } \\
\text { Toro }\end{array}$ & $\mathrm{P}$ & $\mathrm{P}$ & $\mathrm{P}$ \\
\hline 24 & $\begin{array}{l}\text { Cia. Cervecerías } \\
\text { Unidas }\end{array}$ & $\mathrm{P}$ & $\mathrm{P}$ & $\mathrm{P}$ & 59 & $\begin{array}{l}\text { Instituto de } \\
\text { Diagnóstico }\end{array}$ & $\mathrm{P}$ & $\mathrm{P}$ & $\mathrm{P}$ & 94 & $\begin{array}{l}\text { Viña San Pedro } \\
\text { Tarapacá }\end{array}$ & $\mathrm{P}$ & $\mathrm{P}$ & $\mathrm{P}$ \\
\hline 25 & $\begin{array}{l}\text { Cía. Chilena de } \\
\text { Fósforos }\end{array}$ & $\mathrm{P}$ & $\mathrm{P}$ & $\mathrm{P}$ & 60 & Invercap & $\mathrm{P}$ & P & P & 95 & Walmart & $\mathrm{O}$ & $\mathrm{P}$ & $\mathrm{O}$ \\
\hline 26 & $\begin{array}{l}\text { Cía. Electro } \\
\text { Metalúrgica }\end{array}$ & $\mathrm{P}$ & $\mathrm{P}$ & $\mathrm{O}$ & 61 & Invermar & $\mathrm{P}$ & P & $\mathrm{P}$ & 96 & Watts & $\mathrm{P}$ & $\mathrm{P}$ & $\mathrm{P}$ \\
\hline 27 & $\begin{array}{l}\text { Cía. Gral. de } \\
\text { Electricidad }\end{array}$ & $\mathrm{P}$ & $\mathrm{P}$ & $\mathrm{P}$ & 62 & $\begin{array}{l}\text { Inv. Aguas } \\
\text { Metropoli- } \\
\text { tanas }\end{array}$ & $\mathrm{P}$ & P & $\mathrm{P}$ & 97 & $\begin{array}{l}\text { Zona Franca de } \\
\text { Iquique }\end{array}$ & $\mathrm{P}$ & P & $\mathrm{P}$ \\
\hline 28 & $\begin{array}{l}\text { Cía. Ind. El } \\
\text { Volcán }\end{array}$ & $\mathrm{P}$ & $\mathrm{P}$ & $\mathrm{P}$ & 63 & $\begin{array}{l}\text { Inv. La Cons- } \\
\text { trucción }\end{array}$ & $\mathrm{P}$ & $\mathrm{P}$ & $\mathrm{P}$ & & & & & \\
\hline 29 & $\begin{array}{l}\text { Cía. Pesquera } \\
\text { Camanchaca }\end{array}$ & $\mathrm{P}$ & $\mathrm{P}$ & $\mathrm{P}$ & 64 & Invexans & $\mathrm{P}$ & $\mathrm{O}$ & $\mathrm{P}$ & & & & & \\
\hline 30 & $\begin{array}{l}\text { Cía. Sudamerica- } \\
\text { na de Vapores }\end{array}$ & $\mathrm{P}$ & $\mathrm{P}$ & $\mathrm{P}$ & 65 & Inv. Tricahue & $\mathrm{P}$ & $\mathrm{P}$ & $\mathrm{P}$ & & & & & \\
\hline 31 & Cía. Cic & $\mathrm{P}$ & $\mathrm{P}$ & $\mathrm{P}$ & 66 & $\begin{array}{l}\text { Lab. Andró- } \\
\text { maco }\end{array}$ & $\mathrm{P}$ & $\mathrm{P}$ & $\mathrm{O}$ & & & & & \\
\hline 32 & $\begin{array}{l}\text { Cristalerías de } \\
\text { Chile }\end{array}$ & $\mathrm{P}$ & $\mathrm{P}$ & $\mathrm{P}$ & 67 & $\begin{array}{l}\text { Latam Airli- } \\
\text { nes Group }\end{array}$ & $\mathrm{P}$ & $\mathrm{P}$ & $\mathrm{P}$ & & & & & \\
\hline 33 & $\begin{array}{l}\text { Cruz Blanca } \\
\text { Salud }\end{array}$ & $\mathrm{P}$ & $\mathrm{P}$ & $\mathrm{P}$ & 68 & $\begin{array}{l}\text { Marítima de } \\
\text { Inv. }\end{array}$ & $\mathrm{P}$ & $\mathrm{P}$ & $\mathrm{P}$ & & & & & \\
\hline 34 & E.CL & $\mathrm{P}$ & $\mathrm{P}$ & $\mathrm{P}$ & 69 & Masisa & $\mathrm{P}$ & $\mathrm{P}$ & $\mathrm{P}$ & & & & & \\
\hline 35 & $\begin{array}{l}\text { Echeverría Iz- } \\
\text { quierdo }\end{array}$ & $\mathbf{P}$ & $\mathbf{P}$ & $\mathbf{P}$ & 70 & $\begin{array}{l}\text { Minera Valpa- } \\
\text { raíso }\end{array}$ & $\mathrm{P}$ & $\mathrm{P}$ & $\mathrm{P}$ & & & & & \\
\hline
\end{tabular}

\section{ANEXO 2}

Tipos de Respuestas de Sociedades años 2012- 2014.

\section{Clasificación RESPUESTAS}

- $\quad$ En 2014 termina con respuesta afirmativa: Práctica relevante

- Cambio a positivo: en algún año anterior la respuesta no fue positiva, pero terminó siéndolo

- $\quad$ En 2014 termina con respuesta negativa: Práctica irrelevante

- Cambio a negativo: en algún año anterior la respuesta no fue negativa, pero terminó siéndolo 


\section{ANEXO 3}

\section{UTILIZACIÓN MÉTODO DE HIPÓTESIS NULA Y ALTERNATIVA}

La muestra toma en cuenta el método de hipótesis nula y alternativa a partir de la cual se puede determinar si hay significancia estadística para concluir que a partir de la emisión de la norma esta generó un impacto positivo o negativo en las sociedades, tomando en cuenta cada una de las prácticas en particular. Si hay significancia estadística se puede extrapolar a la población en su totalidad.

Test de Hipótesis para la proporción poblacional:

Para realizar un test de hipótesis se comienza por hacer una suposición tentativa del parámetro poblacional (p), a esto se le llama hipótesis nula (. A continuación tenemos otra hipótesis llamada hipótesis alternativa, que plantea lo contrario a la hipótesis nula.

Se busca rechazar la hipótesis nula, de que a nivel poblacional las sociedades el año 2014 que contestaron que sí tienen la práctica sea menor o igual a las que la tenían antes de la norma (2012). Para realizar esta prueba se utilizará un nivel de significancia de $=0,05$. (Se tiene un 95\% de nivel de confianza).

Para calcular el estadístico de prueba se usa la proporción muestral () y su error estándar.

Luego, hay que buscar en la tabla de probabilidad normal estándar el área a la izquierda del estadístico de prueba $(z)$. Luego para determinar si se rechaza la hipótesis nula se utiliza el método del valor-p (1- área a la izquierda de z). Se rechaza la hipótesis nula cuando valor-p es menor al nivel de significancia.

\section{BIBLIOGRAFÍA CITADA}

Alcalde, Enrique (2013): "Las Responsabilidades de los Directores de Sociedades Anónimas" (Santiago, Ediciones UC, primera edición).

BarClay, Kathleen (2015): "Gobierno corporativo: rol fundamental". Disponible en: https://www.df.cl/noticias/opinion/columnistas/kathleen-barclay/gobierno-corporativorol-fundamental/2015-03-30/200850.html. Fecha de consulta: 27 de Julio de 2017.

Black, Bernard S., De Carvalho, Antonio Gledson y Gorga, Erica (2012): "What Matters and for Which Firms for Corporate Governance in Emerging Markets? Evidence from Brazil (and Other BRIK Countries)" 18 Journal of Corporate Finance: pp. 934-952.

Black, Bernard S. y Kraakman, Reinier (1996): "A Self-Enforcing Model of Corporate Law", Harvard Law Review, vol. 109, No 8: pp. 1911-1982.

Camus, Juan Andrés (2015): Exposición Jornada CGC UC 2015. Disponible en: http:// www.bolsadesantiago.com/Biblioteca\%20BCS/Charla\%20Gobiernos\%20Corporativos,\%20Juan\%20Andr\%C3\%A9s\%20Camus.pdf. Fecha de consulta: 27 de Julio de 2017.

Clarke, Thomas (2011): "Corporate Governance Causes of the Global Financial Crisis", en Sun, William (edit.), Corporate Governance and the Global Financial Crisis: International Perspectives (Nueva York, Cambridge University Press) pp. 28-49. 
Coglianese, Cary, Healey, Thomas J., Keating, Elizabeth K. y Michael. Michael L. (2004): "The Role of Government in Corporate Governance," NYUJL \& Business, vol. 1: p. 219-239.

Coloma, Fernando (2012): Exposición en Jornada CGC UC 2012. Disponible en: https:// www.svs.cl/portal/prensa/604/articles-15490_doc_pdf.pdf. Fecha de consulta: 27 de Julio de 2017.

Coombes, Paul y Wong Simon Chui-Yin, (2004): "Why Codes of Governance Work", McKinsey Quarterly, No 2: pp. 48-53.

Corbo, Vittorio y Schmidt-Hebbel, Klaus (2003): "Macroeconomic Effects of Pension Reform in Chile," in Pension Reforms: Results and Challenges, International Federation of Pension Fund Administrators, FIAP, Santiago, Chile, pp.241-329. Disponible en: http://www.scielo.cl/scielo.php?script=sci_nlinks\&ref=1814106\&pid=S07176821200600010000100036\&lng=es. Fecha de consulta: 27 de Julio de 2017.

Enriques, Lucas, Hansmann, Henry y Kraakman, Rainer (2009): "The Basic Governance Structure: The Interests of Shareholders as a Class", en KRAAKMAN, Rainer et al. (edit.), The Anatomy of Corporate Law (Oxford University Press) pp. 55-82.

García, Rodrigo (2014): "Norma de Información sobre Prácticas de Gobierno Corporativo" disponible en página web SVS https://www.svs.cl/portal/publicaciones/610/articles-16379_doc_pdf.pdf.

Fecha de consulta: 27 de Julio 2017.

Haskovec, Nolan (2012): "Codes of Corporate Governance”, Working Paper, Millstein Center for Corporate Governance and Performance. Disponible en: http://web.law. columbia.edu/sites/default/files/microsites/millstein-center/Codes\%20of\%20Corporate\%20Governance_Yale_053112.pdf. Fecha de consulta: 27 de Julio de 2017.

Hertig, Gerard (2005): “On-going Board Reforms: One Size Fits All and Regulatory Capture”, Oxford Review of Economic Policy, vol. 21, Issue 2: pp. 269-282.

Hormazábal, Soledad (2010): "Corporate Governance and Pension Fund Administrators: The Chilean Case, Economic Research Department No 10/06. Disponible en https:// www.bbvaresearch.com/wp-content/uploads/migrados/WP_1006_tcm348-220369.pdf. Fecha de consulta: 27 de Julio de 2017.

Irwin, Steven, Lane, Scott, Mandelson, Carolyn y Tighe, Tara (2012): "Self-Regulation of the American Retail Securities Markets - An Oxymoron for What is Best for Investors?”, U. of Pennsylvania Journal of Business Law, vol. 14:4 : pp. 1055-1084.

La Tercera (21/04/2013): "Las sociedades anónimas formalizan cambios a gobiernos corporativos". Disponible en: http://www.latercera.com/noticia/las-sociedades-anonimasformalizan-cambios-a-gobiernos-corporativos/.

LeFort, Fernando y GonZÁlez, Rodrigo (2008): "Hacia un mejor gobierno corporativo en Chile", Revista ABANTE, vol. 11, No1: pp. 19-45.

Lefort, Fernando y Walker, Eduardo (2000): "The effects of economic and political shocks on corporate governance systems in Chile", Revista ABANTE, vol. 2, No 2: pp. 183-206.

Lefort, Fernando y Walker, Eduardo (2002): "Pension Reform and Capital Markets: Are There Any (Hard) Links?", Revista ABANTE, vol. 5, No 2: pp. 77-149. 
LEFORT, Fernando Y WalKer, Eduardo (2005): “Ownership structure and corporate governance in Latin America”, Revista ABANTE, vol. 8, No1: pp. 55-84.

Mckinsey E ICARE (2007): "Potenciando el Gobierno Corporativo de las Empresas en Chile". Disponible en: https://www.sbif.cl/sbifweb/servlet/ Ebook?CTR=XXXXXXXXXXXXXXX\&ID_EBOOK=L3062\&position=0. Fecha de consulta: 27 de Julio de 2017.

Núñez, David y Pardow, Diego (2010): “¿Por qué no demandan los accionistas? el problema de las costas en la acción derivativa”, Revista Estudios Públicos, No 118: pp. 229-282

Organización para la Cooperación y Desarrollo Económico (OECD) (2011): “Corporate Governance in Chile 2010" Corporate Governance, OECD Publishing, Paris.

Organización para la Cooperación y Desarrollo Económico (OECD) (2015): Corporate Governance Factbook 2015. Disponible en http://www.oecd.org/daf/ca/corporategovernance-factbook.htm. Fecha de consulta: 27 de Julio de 2017.

Pavez, Carlos (2014): "Los nuevos desafíos del gobierno corporativo". Disponible en: http://www.svs.cl/portal/prensa/604/articles-16666_doc_pdf.pdf. Fecha de consulta: 27 de Julio de 2017.

Pez-Ares, Cándido (2004): "El gobierno corporativo como estrategia de creación de valor, Indret”, Working Paper $\mathrm{N}^{\circ}$ 182. Disponible en: http://www.indret.com/pdf/182_ es.pdf. Fecha de consulta: 27 de Julio de 2017.

Wilenmann, Javier (edit.) (2010): “Gobiernos Corporativos. Aspectos esenciales de las reformas a su regulación" (Santiago, Thomson Reuters).

World BANK, (2003): Report of Standard and Codes (ROSC), corporate governance country assessment. Disponible en http://documents.worldbank.org/curated/ en/769111468022464847/Chile-Report-on-the-Observance-of-Standards-and-CodesROSC-corporate-governance-country-assessment Fecha de Consulta 27 de Julio de 2017.

Young, Michael. N., Peng, Mike W., Ahlstrom, David, Bruton, Garry. D., y Jiang, Yi (2008): "Corporate governance in emerging economies: A review of the principal-principal perspective", Journal of Management Studies, vol. 45, No1: pp. 196-220.

Zegers, Matías y Consiglio, Josefina (2013): "Gobierno Corporativo en Latinoamérica: Un análisis comparativo entre Brasil, Chile, Colombia y México". Disponible en http:// www.centrobuengobierno.ie.edu/wp-content/uploads/sites/87/2013/11/GobiernoCorporativoEnLatiniamericaUnAnalisisComparativoEntreBrasilChileColombiaMexico.pdf. Fecha de consulta: 2 de Septiembre de 2016.

UK Financial Reporting Council, (2012): "What constitutes an explanation under comply or explain? Report of discussion between companies and investors". Disponible en: https://www.frc.org.uk/Our-Work/Publications/Corporate-Governance/What-constitutes-an-explanation-under-comply-or-ex.pdf. Fecha de consulta: 27 de Julio de 2017.

\section{NORMAS CITADAS}

Dutch Corporate Governance Code (2009). Disponible en HTTP://WWW.COMMISSIECORPORATEGOVERNANCE.NL/DUTCH-CORPORATE-GOVERNANCECODE. 
Historia DE LA LEY No 20.382 (20/10/2009). Introduce perfeccionamientos a la normativa que regula los gobiernos corporativos de las empresas.

Malasyan Code on Corporate Governance (2012). Disponible en http://www.sc.com. my/wp-content/uploads/eng/html/cg/cg2012.pdf.

SVS (2015). Informe Final Fortalecimiento de Estándares de Gobierno Corporativo de las Sociedades Anónimas Abiertas. NCG No385 emitida por la SVS.

Swedish Corporate Governance Code (2015). Disponible en HTTP://WWW.CORPORATEGOVERNANCEBOARD.SE/THE-CODE/CURRENT-CODE.

UE COMMISSION RECOMMENDATION of 9 April 2014 on the quality of corporate governance reporting (comply or explain). Disponible en: http://eur-lex.europa.eu/legal-content/ EN/TXT/?uri=CELEX:32014H0208.

UK Corporate Governance Code (2014). Disponible en https://www.frc.org.uk/OurWork/Publications/Corporate-Governance/UK-Corporate-Governance-Code-2014.pdf. 